ARTICLE

\title{
Antibody-guided in vivo imaging of Aspergillus fumigatus lung infections during antifungal azole treatment
}

Sophie Hennebergㄹ, Anja Hasenberg (10 1, Andreas Maurer (10 2, Franziska Neumann', Lea Bornemann (1) 1, Irene Gonzalez-Menendez ${ }^{3}$, Andreas Kraus ${ }^{1}$, Mike Hasenberg ${ }^{1}$, Christopher R. Thornton (1) 4, Bernd J. Pichler ${ }^{2}$, Matthias Gunzer (iD) ${ }^{1,5} \otimes$ \& Nicolas Beziere (iD ${ }^{2 凶}$

Invasive pulmonary aspergillosis (IPA) is a life-threatening lung disease of immunocompromised humans, caused by the opportunistic fungal pathogen Aspergillus fumigatus. Inadequacies in current diagnostic procedures mean that early diagnosis of the disease, critical to patient survival, remains a major clinical challenge, and is leading to the empiric use of antifungal drugs and emergence of azole resistance. A non-invasive procedure that allows both unambiguous detection of IPA and its response to azole treatment is therefore needed. Here, we show that a humanised Aspergillus-specific monoclonal antibody, dual labelled with a radionuclide and fluorophore, can be used in immunoPET/MRI in vivo in a neutropenic mouse model and 3D light sheet fluorescence microscopy ex vivo in the infected mouse lungs to quantify early A. fumigatus lung infections and to monitor the efficacy of azole therapy. Our antibody-guided approach reveals that early drug intervention is critical to prevent complete invasion of the lungs by the fungus, and demonstrates the power of molecular imaging as a non-invasive procedure for tracking IPA in vivo.

\footnotetext{
${ }^{1}$ Institute for Experimental Immunology and Imaging, University Hospital, University of Duisburg-Essen, Essen, Germany. ${ }^{2}$ Werner Siemens Imaging Center, Department of Preclinical Imaging and Radiopharmacy, Eberhard Karls University, Tübingen, Germany. ${ }^{3}$ Department of Pathology and Neuropathology, Eberhard Karls University, Tübingen, Germany. ${ }^{4}$ ISCA Diagnostics Ltd. and Biosciences, College of Life and Environmental Sciences, University of Exeter, Exeter, UK.

${ }^{5}$ Leibniz-Institut für Analytische Wissenschaften ISAS -e.V, Dortmund, Germany. ${ }^{凶}$ email: matthias.gunzer@uni-due.de; nicolas.beziere@med.uni-tuebingen.de
} 
spergillus fumigatus is an abundant environmental mold, with air-borne spores that are frequently inhaled. The fungus is an opportunistic pathogen, and while the immune system of healthy individuals is highly effective at eliminating infective spores from the lung, patients with impaired immunity, especially those with hematological malignancies, prolonged neutropenia, or recipients of hematopoietic stem cell or solid organ transplants, are at elevated risk of developing invasive pulmonary aspergillosis (IPA), a rapidly progressive and often fatal lung disease caused by germinated spores of the pathogen. The disease is also increasingly reported in patients with underlying respiratory diseases such as severe asthma and COPD, and as co-infections in patients with severe influenza and with Covid-19 coronavirus $^{1-3}$, regardless of their immune system status $^{4,5}$. The high mortality rate of $\operatorname{IPA}^{6}$ is exacerbated by the lack of rapid, specific and sensitive diagnostic tests. Since the symptoms of the disease (fever and chills, haemoptysis, shortness of breath, chest pain, headaches) are nonspecific, diagnosis relies on culture of the pathogen from invasive biopsy ${ }^{7}$, or detection of biomarkers in serum or in bronchoalveolar lavage fluid (BALf) recovered during invasive bronchoscopy ${ }^{8}$. Attempts have been made to improve noninvasive diagnosis using computed tomography $(\mathrm{CT})^{8}$ or magnetic resonance imaging (MRI) ${ }^{9}$ of the chest, but radiological indicators of infection are not pathognomonic for IPA. Despite this, a radiological abnormality in a chest-CT is used in many centers as a trigger for initiating antifungal drug treatment in a febrile patient unresponsive to antibiotics ${ }^{10}$. Inappropriate or delayed treatment of IPA, driven by imprecise diagnostic procedures, negatively impacts on patient morbidity and mortality, and is contributing to the emergence of azole resistance in clinical strains of $A$. fumigatus ${ }^{11,12}$. Therefore, improvements in IPA diagnosis are needed that allow noninvasive detection of lung infection in vivo, and enable monitoring of disease responsiveness to antifungal drug treatments.

The use of targeted contrast agents in molecular imaging procedures such as positron emission tomography (PET) or single photon emission computed tomography (SPECT) holds enormous promise in addressing this unmet need ${ }^{13,14}$. While PET has proven its imaging capabilities, leading to its widespread use in oncology, only very few studies have tried to capitalize on its potentially high sensitivity and selectivity for infectious disease diagnosis beyond $\left[{ }^{18} \mathrm{~F}\right] \mathrm{FDG}$. For invasive mycoses, radiolabeled antifungal drugs ${ }^{15}$ or fungal siderophores ${ }^{16,17}$ have been attempted, with mixed preclinical success, but their translation to the clinic appears problematic due to their lack of specificity. This limitation is overcome by the use of radiolabeled monoclonal antibodies, whose specificity allows targeted detection of individual pathogens when applied in immunoPET. Recently, we have focused on an Aspergillus-specific mAb, JF5 ${ }^{18}$, which binds to an antigen expressed on the surface of actively growing hyphae of $A$. fumigatus. While initial investigations using ${ }^{64} \mathrm{Cu}$-radiolabeled murine JF5 showed promising results for in vivo imaging of IPA in a mouse model of the disease ${ }^{19}$, further improvements in JF5guided PET/MR imaging were met through its humanization ${ }^{20}$, enabling translation of the technology to the clinical setting.

An important but unexplored aspect of molecular imaging is its use as a treatment monitoring tool for invasive fungal diseases. While attempts have been made to use circulating biomarkers to monitor responsiveness of IPA to antifungal treatment ${ }^{21}$, it is well-documented that the sensitivities of diagnostic biomarker tests for the disease, such as the Aspergillus galactomannan (GM) ELISA and fungal $\beta$-D-glucan assay, are markedly influenced by mold-active azoles such as voriconazole (VCZ), both in model systems and in humans ${ }^{22,23}$. Furthermore, there is no data currently available on the relationship between fungal biomass in the lung and amounts of circulating biomarkers in serum or BALf, and so it is not possible to determine unequivocally whether lung infection has been resolved without a visual appraisal using nonspecific chest-CT.

A key step to using immunoPET/MRI as a treatment monitoring tool is to establish whether it is sufficiently accurate to allow changes in Aspergillus load in the lung to be quantified in response to antifungal drug treatment. To this end, we set out here to (1) establish whether accumulation in the lung of the humanized JF5 (hJF5) radiotracer during A. fumigatus infection of neutropenic mice is directly related to fungal load, and (2) whether changes in the fungal load in the lung coincident with VCZ treatment can be monitored quantitatively in vivo. To achieve these goals, we developed a dual-labeled hJF5 variant bearing both a radionuclide and a fluorophore, $\left[{ }^{64} \mathrm{Cu}\right] \mathrm{Cu}$ NODAGA-hJF5-DyLight650 (hereafter ${ }^{64} \mathrm{Cu}$-hJF5-DyLight650), which allowed us to co-localize and to co-quantify the pathogen and tracer in the lung both in vivo using broad resolution immunoPET/MRI, and ex vivo using high-resolution 3D light sheet fluorescence microscopy. Using this approach, we show that immunoPET/MRI can be used to monitor disease progression during treatment with an azole drug, and that early intervention with the drug is critical to preventing A. fumigatus infection.

\section{Results}

Characterization of the dual-labeled ${ }^{64} \mathrm{Cu}-\mathrm{hJF} 5-\mathrm{DyLight} 650$ antibody. The elution profile of the double-conjugated and radiolabeled antibody showed a single peak corresponding to the molecular weight of the antibody, with no detectable aggregates and only a small $(<5 \%)$ dimer peak (Supplementary Fig. 1). To exclude detrimental effects of the chelator and fluorophore conjugation to antigen binding, the reactivity of the doubleconjugated antibody was determined by ELISA using serial dilutions of the respective antibodies (Supplementary Fig. 2). We found only slightly reduced antigen binding of the antibody after single (NODAGA) and double (NODAGA and DyLight) conjugations, compared to the unconjugated antibody. Fitting of the binding curves revealed apparent Kd values of $67 \mathrm{pM}$ (unmodified antibody), $59 \mathrm{pM}$ (NODAGA-conjugated), and $112 \mathrm{pM}$ (double-conjugated).

Light sheet fluorescence microscopy of $A$. fumigatus in healthy and neutropenic mice. To visualize the proliferation of $A$. fumigatus $^{\text {tdTomato }}$ in healthy and neutropenic animals, their lungs were removed $30 \mathrm{~min}, 6,24$, and $48 \mathrm{~h}$ postinfection. Following fixation and clearing, the lungs were imaged using Light Sheet Fluorescence Microscopy with the fungus being detectable via the endogenous tdTomato fluorescence signal (Fig. 1a). This revealed that in both immunosuppressed (neutropenic) and immunocompetent animals, there was patchy distribution of the pathogen $30 \mathrm{~min}$ after administration of the spore inoculum (Fig. 1b). Infestation of the lungs of neutropenic mice had occurred by $6 \mathrm{~h}$ p.i., with further proliferation of the pathogen thereafter until termination of the experiment. In comparison, healthy animals receiving the same spore inoculum showed a markedly reduced proliferation of the pathogen during the first $6 \mathrm{~h}$ postinfection, and a complete cessation by $48 \mathrm{~h}$ (Fig. 1b). High-resolution multiphoton microscopy of the same samples showed that the neutrophil depletion permitted substantial hyphal outgrowth by $24 \mathrm{~h}$ which was further increased by $48 \mathrm{~h}$. In contrast, the presence of neutrophils completely blocked hyphal development, and led to total recovery of previously infected lung tissues, such that by $48 \mathrm{~h}$ it was morphologically indistinguishable from the noninfected lung (Fig. 1c).

Quantification showed that while the initial loads of A. fumigatus were comparable at the earliest time point (30 min p.i.), differences 
a
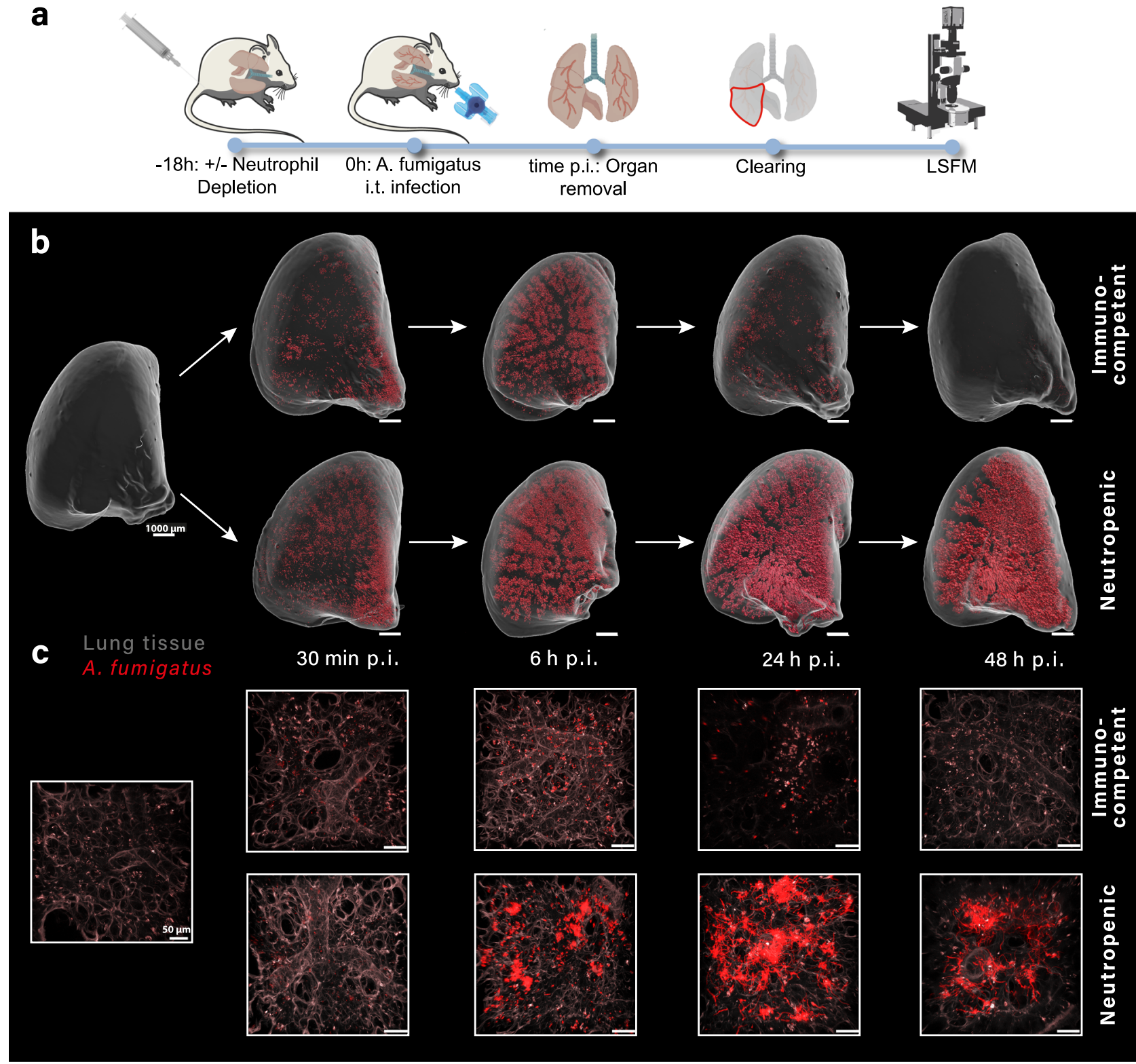

d

Progress of fungal load over time

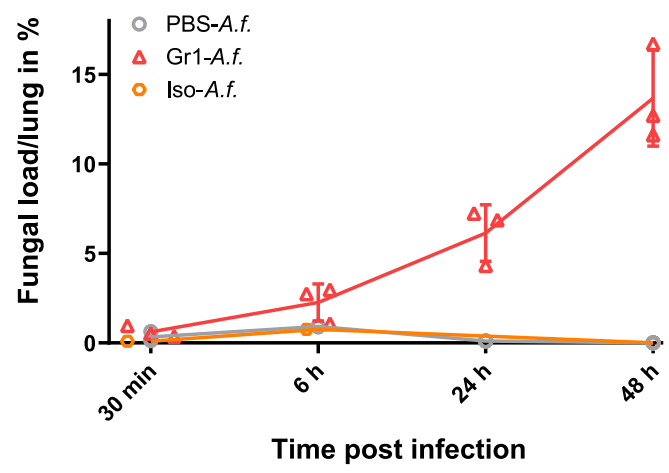

in loads between healthy and neutropenic mice were evident as early as $6 \mathrm{~h}$ p.i., and continued to rise over the period of observation (Fig. 1d). Hence, a functional neutrophil response controls fungal spread within the first hours of infection and effectively suppresses hyphal development.
In vivo immunoPET/MR of $A$. fumigatus infection and treatment monitoring. In previous studies, we showed the potential of the murine mAb JF5 and its humanized counterpart hJF5 ${ }^{19,20}$ to detect A. fumigatus lung infection in vivo using PET/MR. To determine whether this approach could be applied to treatment 
Fig. 1 Development of invasive pulmonary aspergillosis under healthy and immunosuppressed conditions. a Schematic experimental workflow. Neutropenic groups were depleted with $\mathrm{Gr}-1$ antibody $18 \mathrm{~h}$ prior to intratracheal (i.t.) inoculation with Aspergillus fumigatus ${ }^{\text {tdTomato }}$ conidia. Lungs were subsequently removed at different time postinfection (p.i.), cleared and imaged using light sheet fluorescence microscopy (LSFM). b Lung tissue (gray) and fungal biomass (red) is depicted for time points $0,0.5,6,24$, and $48 \mathrm{~h}$ during disease progression. Images were constructed using surface tool from Imaris. Scale bar $=1 \mathrm{~mm}$. c Magnified two-photon microscopy images of the same lungs as in $\mathbf{b}$, with lung tissue (gray), and fungal biomass (red). Scale bar $=50$ $\mu \mathrm{m}$. d Plot of fungal load per whole lung lobe for the different treatments over time. Sham-depleted, Aspergillus fumigatus (A.f.) infected group (PBS-A.f., gray circle, $n=2$ per time point), Gr-1 neutrophil-depleted and infected group (Gr1-A.f., red triangles, $n=3$ per time point), isotype-depleted and infected group (Iso-A.f., orange squares, $n=1$ per time point). Results are plotted as individual values with average and standard deviation where applicable.

monitoring, we investigated the growth of $A$. fumigatus ${ }^{\text {tdTomato }}$ in the lungs of healthy or neutrophil-depleted mice in response to treatment with VCZ, a triazole drug used in humans for primary therapy of IPA ${ }^{24}$. We first determined the VCZ susceptibility of our A. fumigatus strains (the wild-type strain ATCC 46645 and the genetically modified derivative A. fumigatus $^{\text {tdTomato }}$. Minimum inhibitory concentration (MIC) determination for VCZ by visual reading, as suggested in the EUCAST guidelines ${ }^{25}$, resulted in $0.5 \mu \mathrm{g} / \mathrm{ml}$ for ATCC 46645 as well as for A. fumigatus ${ }^{\text {tdTomato }}$ strain (Supplementary Fig. 3 and Supplementary Table 1), similar to published data 25 . To corroborate these results with a quantitative reading method we analyzed the 96-well plates spectrophotometrically following the suggestion of Meletiadis et al. ${ }^{26}$. The results of the spectrophotometrical analysis yielded a VCZ MIC of $0.5 \mu \mathrm{g} / \mathrm{ml}$ for ATCC 46645 and of $0.25 \mu \mathrm{g} / \mathrm{ml}$ for strain A. fumigatus ${ }^{\text {tdTomato }}$ (Supplementary Fig. 4 and Supplementary Table 2). These confirmed our previous findings using visual appraisals, and are consistent with the MIC range published in the works of Lass-Flörl and Meletiadis et al. In in vivo experiments, we first tested i.v. administration of VCZ $24 \mathrm{~h}$ postinfection and tracer injection (Fig. 2a), the time period corresponding to widespread colonization of the neutropenic lung (Fig. 1b). Images acquired 24 and $48 \mathrm{~h}$ after ${ }^{64} \mathrm{Cu}-\mathrm{hJF} 5$ and inoculum injection, showed no differences in the qualitative distribution of the radiotracer in the lungs of animals with VCZ treatment and those without (Fig. 2b). Indeed, significant accumulation was seen in the lungs of both groups of animals due to specific binding of the antibody tracer to its hyphal-bound antigen, and in the spleen due to soluble circulating antigen, a phenomenon reported previously ${ }^{19,20}$. As expected, a ${ }^{64} \mathrm{Cu}$ signal could also been seen in the heart, indicating prolonged circulation of the radiotracer. Quantification of the in vivo ${ }^{64} \mathrm{Cu}$ PET signal in the lungs, expressed as \%ID/cc (Fig. 2c), confirmed the distributions seen qualitatively (Fig. 2b). No significant differences in tracer uptake in the lungs were found between VCZ-treated and non-treated animals at $24 \mathrm{~h}(10.0 \pm 2.0 \% \mathrm{ID} / \mathrm{cc}$ and $9.9 \pm 1.7 \% \mathrm{ID} / \mathrm{cc}$, respectively) and $48 \mathrm{~h}(11.1 \pm 1.7 \% \mathrm{ID} / \mathrm{cc}$ and $12.9 \pm 2.7 \% \mathrm{ID} / \mathrm{cc}$, respectively). At $48 \mathrm{~h}$, a significant increase in lung uptake of ${ }^{64} \mathrm{Cu}-\mathrm{h} J \mathrm{~F} 5$ could be seen in both groups compared to the control animals $(6.5 \pm 1.7 \% \mathrm{ID} / \mathrm{cc})$ and, while the lung PET signal remained stable through time in control animals, the signal in infected animals increased significantly over the $48 \mathrm{~h}$ period, reaching a contrast ratio of $\sim 2$. Ex vivo biodistribution analysis of the tracer in different organs at $48 \mathrm{~h}$ (Fig. 2d) after perfusion by $0.4 \%$ PFA showed no significant differences in lung uptake between VCZtreated and non-treated animals $(38.4 \pm 8.9 \% \mathrm{ID} / \mathrm{g}$ and $41 \pm 18 \%$ $\mathrm{ID} / \mathrm{g}$, respectively), but with both significantly greater compared to control (uninfected) animals $(3.9 \pm 1.2 \% \mathrm{ID} / \mathrm{g})$. Although no differences could be seen in heart or muscle uptake between groups, differences in the accumulation of ${ }^{64} \mathrm{Cu}-\mathrm{hJF} 5$ could be seen in the spleens of control and infected animals, with infected animals displaying an approximate twofold increase in uptake regardless of VCZ treatment. The circulating blood levels of the ${ }^{64} \mathrm{Cu}-\mathrm{h}$ JF5 tracer, obtained from blood sampling performed directly before animal perfusion, were similar in the infected cohorts $(31.9 \pm 5.8 \% \mathrm{ID} / \mathrm{cc}$ and $36.4 \pm 2.8 \% \mathrm{ID} / \mathrm{cc}$ in untreated and azole-treated groups, respectively), which was higher than in the control group $(19.7 \pm 2.6 \% \mathrm{ID} / \mathrm{cc})$ indicating the long half-life of the full-length IgG in the bloodstream, but also possibly due to binding of the radiolabeled antibody to circulating antigen. Collectively, these data show that treatment with VCZ following establishment of lung infection by A. fumigatus, has no measurable effect on progression of disease in the neutropenic lung within the timeframe of the experiment.

Dual-label in vivo immunoPET and ex vivo LSFM. The lack of effect of VCZ led us to investigate whether the macroscopic resolution of $\mathrm{PET} / \mathrm{MR}$ was insufficient to discriminate subtle differences in fungal burden consequent with antifungal treatment. We therefore set out to investigate the correlation between tracer accumulation and fungal burden in the lung using a combined PET/MR and LSFM imaging approach. To this end, we developed a dual-labeled derivative of hJF5, with ${ }^{64} \mathrm{Cu}$ as the radioactive PET reporter, and DyLight650 as a fluorescent reporter for optical imaging. After infecting neutropenic mice with $A$. fumigatus ${ }^{\text {tdTomato }}$ and immediately injecting ${ }^{64} \mathrm{Cu}-\mathrm{hJF5}$ DyLight650, we determined whether VCZ administered $24 \mathrm{~h}$ after infection had an effect on the spatial distribution of the antibody $48 \mathrm{~h}$ after infection, and its co-localization with the fungus in infection sites at a macroscopic and microscopic level (Fig. 3a). Imaging the same lungs with both in vivo immunoPET/MR and ex vivo LSFM (Fig. 3b, c) revealed, qualitatively, complete overlap between fungal growth (depicted by red tdTomato fluorescence) (Fig. 3c), hJF5 labeling of hyphal antigen via the green DyLight650 fluorophore (Fig. 3c), and macroscopic distribution in vivo via the radionuclide ${ }^{64} \mathrm{Cu}$ in immunoPET/MR (Fig. 3b). Confocal microscopy of the ${ }^{64} \mathrm{Cu}-\mathrm{hJF} 5-\mathrm{DyLight} 650$ molecule and tdTomato fluorescence further showed co-localization of fungal foci and the antibody at the microscopic level (Fig. 3d).

In vivo and ex vivo volumetric quantification of fungal invasion. In order to quantify fungal load in the lungs in vivo, we investigated how thresholding of the PET signal at different radiotracer accumulation values might enable accurate measurements of the volume occupied by the pathogen to be made. The ratio of the infection volume, as measured in PET, to the overall lung volume measured in MRI is shown in Fig. 4a. While a low threshold of $5 \% \mathrm{ID} / \mathrm{cc}$, under the average $\% \mathrm{ID} / \mathrm{cc}$ in healthy animals, provided a significant difference between healthy controls and diseased animals, it nevertheless gave a strong false negative result, with $\sim 50 \%$ of lungs supposedly infected in control animals. The variability was also high, since the threshold value was close to the background level. In contrast, a threshold of $10 \% \mathrm{ID} / \mathrm{cc}$, selected to be significantly above the average PET tracer accumulation of a healthy lung at $48 \mathrm{~h}(6.5 \pm 1.7 \% \mathrm{ID} / \mathrm{cc})$, showed a significant difference between control and infected animals $(3.2 \pm$ $4.5 \%$ compared to $61 \pm 4.5 \%$; $p<0.005)$. However, VCZ-treated animals did not show any difference when compared to non- 

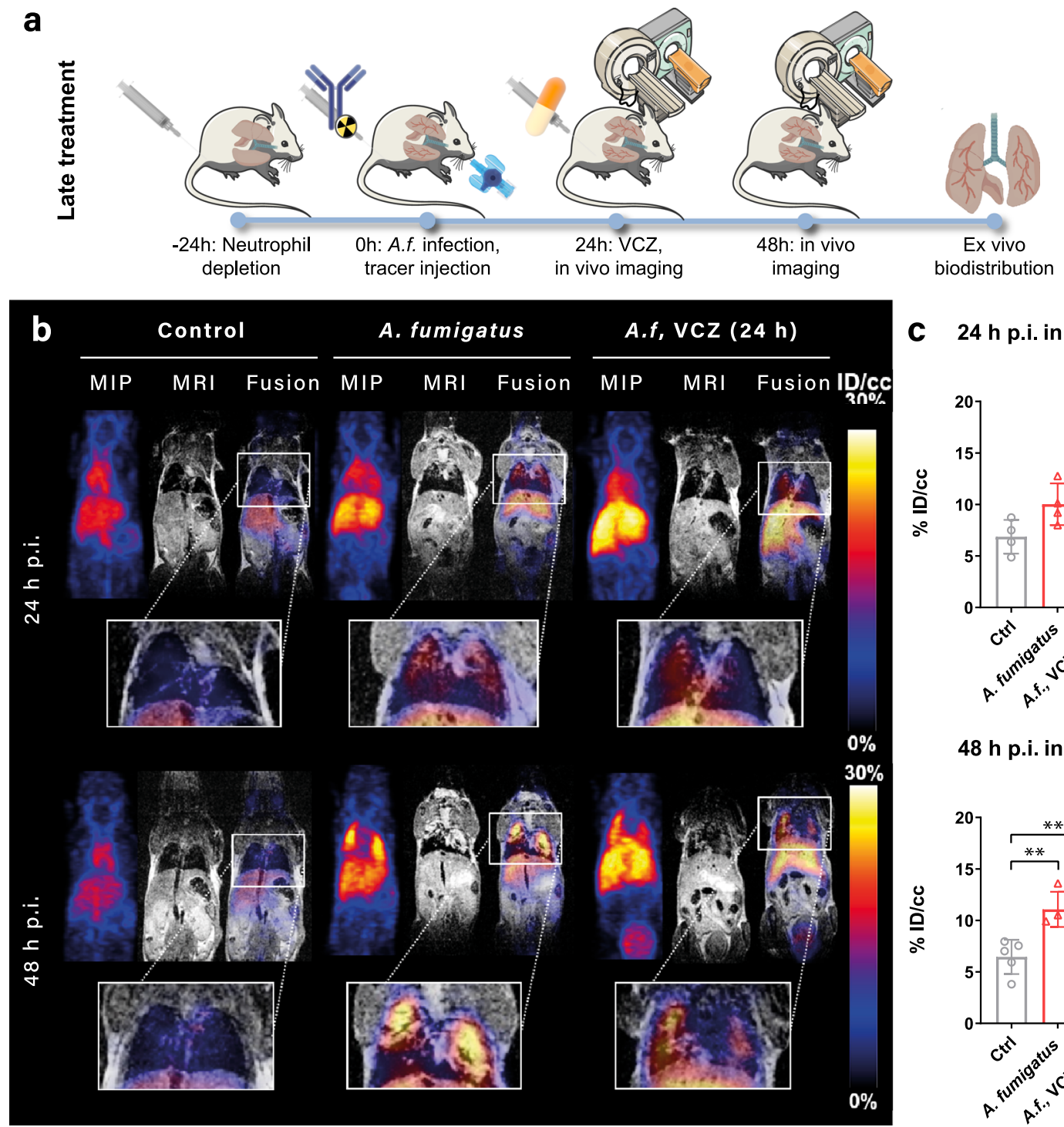

C 24 h p.i. in vivo PET

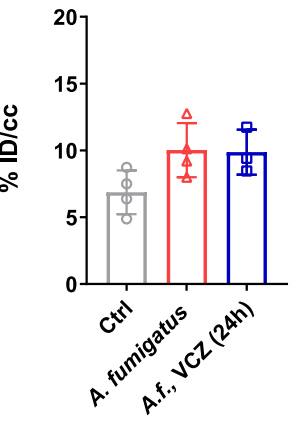

\section{$48 \mathrm{~h}$ p.i. in vivo $\mathrm{PET}$}

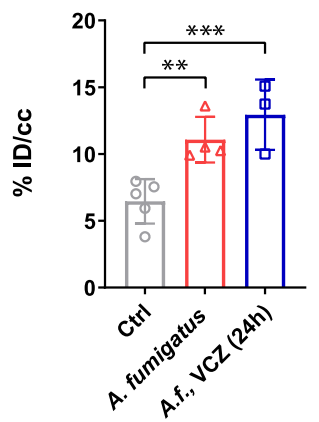

d

ex vivo Biodistribution

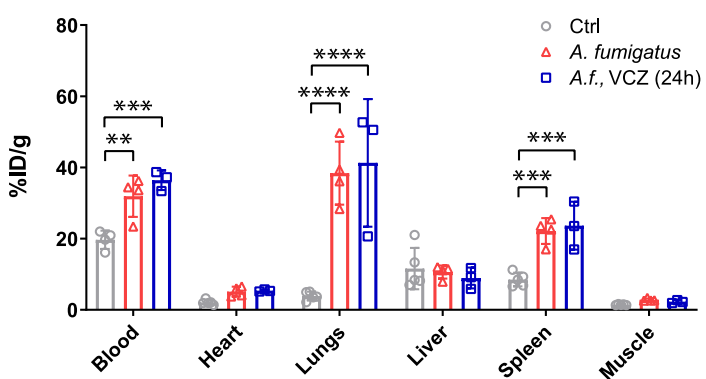

treated animals, with an infected volume of $54 \pm 8 \%$. A further increase in threshold volume to $15 \% \mathrm{ID} / \mathrm{cc}$, selected to match the maximal PET signal obtained from healthy lung tissue in vivo $(14.3 \pm 1.6 \% \mathrm{ID} / \mathrm{cc}$ on average) provided an infected volume of $0 \%$ for control animals with no difference between non-treated and treated infected animals ( $22 \pm 5 \%$ and $21 \pm 10 \%$, respectively). An even higher threshold of $20 \% \mathrm{ID} / \mathrm{cc}$ provided negligible infection volumes of $<5 \%$ in infected animals but did not yield significant differences between groups. In addition, we performed a receiver operating characteristic analysis (Supplementary Fig. 5). This showed that 10 and $15 \% \mathrm{ID} / \mathrm{cc}$ were optimal thresholding values allowing differentiation between healthy and infected animals with an area under curve (AUC) of 1, whereas the threshold values 5 and $20 \% \mathrm{ID} / \mathrm{cc}$ showed an AUC under 0.9. A similar thresholding approach was applied to LSFM images (Fig. 4b), comparing the volumes occupied by $A$. fumigatus ${ }^{\text {tdTomato. While control animals }}$ did not present any fluorescent signal, similar values for volume occupancy by the pathogen and by the ${ }^{64} \mathrm{Cu}-\mathrm{hJF} 5-$ DyLight 650 tracer were found in infected animals, and in VCZ-treated and non-treated infected animals, with $\sim 20 \%$ of the lung displaying fluorescence signals (and hence active infection). Co-localization of A. fumigatus and hJF5, as measured in LSFM, yielded $~ 75 \%$ 
Fig. 2 ImmuoPET/MR imaging of invasive pulmonary aspergillosis in response to voriconazole (VCZ) treatment. a Experimental workflow depicting neutrophil depletion, Aspergillus fumigatus (A.f.) infection, tracer injection, voriconazole administration (VCZ) and in vivo imaging. $\mathbf{b}$ ImmunoPET/MR images of PBS-deposited animals (Control, left column, $n=4$ ), A. fumigatus-infected animals without VCZ treatment ( $A$. fumigatus, middle column, $n=4$ ), and with late-stage VCZ treatment ( $A . f . V C Z(24 \mathrm{~h}$ ), right column, $n=3$ ). Images acquired $24 \mathrm{~h}$ after inoculation (p.i.) are presented in the top row, the bottom row are images acquired $48 \mathrm{~h}$ p.i.. MIP is a Maximum Intensity Projection of the PET image; MRI is a T2-weighted MRI single slice MRI image; Fusion is a single slice PET image overlaid on the corresponding MRI slice. Magnification of the fusion image in the lung area is displayed below. c Quantification of ${ }^{64} \mathrm{Cu}$-hJF5 accumulation in the lung tissue at $24 \mathrm{~h}$ (top) and $48 \mathrm{~h}$ (bottom) after radiotracer injection in control (Ctrl), infected (A. fumigatus) and voriconazole treated animals (A.f., VCZ (24 h)). $48 \mathrm{~h}$ p.i.: Ctrl vs. A. fumigatus, $p=0.0152 ;$ Ctrl vs. A.f., VCZ (24 h), $p=0.0033$. d Ex vivo Biodistribution data of ${ }^{64} \mathrm{Cu}$-hJF5 in major organs $48 \mathrm{~h}$ after radiotracer injection obtained after perfusion of the animal by $0.4 \%$ PFA. Blood: Ctrl vs. A. fumigatus, $p=0.0032$; Ctrl vs. A.f., VCZ (24 h), $p=0.0002$. Lungs: Ctrl vs. A. fumigatus, $p<0.0001$; Ctrl vs. A.f., VCZ (24 h), $p<0.0001$. Spleen: Ctrl vs. A. fumigatus, $p=0.0005 ; \mathrm{Ctrl}$ vs. A.f., VCZ $(24 \mathrm{~h}), p=0.0004$. Results are plotted as individual values with average and standard deviation and are expressed as percent injected dose per cubic centimeter (\%ID/cc) for PET and injected dose per gram (ID/g) for Biodistribution. $p$ values were generated using one way ANOVA (c) or two way ANOVA (d) with Tukey's multiple comparison test $\left({ }^{\star} p<0.05 ;{ }^{\star \star} p<0.01 ;{ }^{\star \star \star} p<0.001 ;{ }^{\star \star \star \star} p<0.0001\right)$.

correlation in both treated and non-treated groups (Fig. 4c), which was further supported by good correlation between the volume occupied by A. fumigatus in the diseased lung and hJF5DyLight650 accumulation, regardless of VCZ treatment (Fig. 4d). This indicated that DyLight-hJF5 faithfully reports $A$. fumigatus invasion in the organ. When the in vivo immunoPET/ MRI and ex vivo LSFM data were combined with a $15 \% \mathrm{ID} / \mathrm{cc}$ threshold for determination of disease volume (obtained by thresholding based on maximum signal intensity in healthy lungs), correlation between immunoPET/MR and LSFM volumetric findings per animal (Fig. 4e) showed a linear correlation $\left(r^{2}=0.8978\right.$ and a slope of $\left.0.87 \pm 0.17\right)$, which was not the case with a threshold of $10 \%\left(r^{2}=0.1832\right)$. Hence, dual-label imaging with an hJF5-targeted strategy allows precise determination of the extent of fungal loading at a macroscopic and microscopic level.

Early onset therapy is critical for infection control. To treat IPA successfully, it is essential to discover lung infection as early as possible during disease progression, so as to allow timely treatment with mold-active drugs. To compare the effectiveness of early- and late-stage treatment on disease progression, we evaluated animals treated with VCZ at 3 and $24 \mathrm{~h}$ postinfection using immunoPET/ MR. We had already established using LSFM (Fig. 1) that by $6 \mathrm{~h}$ postinfection the pathogen had begun to invade the entire lung, albeit at a low level, while widespread establishment of the pathogen had occurred by $24 \mathrm{~h}$. We therefore chose the time points 3 and 24 $\mathrm{h}$ postinfection as the points at which to administer VCZ, so that we could then determine the effectiveness of early and late antifungal treatment start on disease progression using immunoPET/MR (Fig. 5a). At $24 \mathrm{~h}$ postinfection, immunoPET/MR images of disease progression under the two different VCZ treatment regimens $(3 \mathrm{~h}$ $+24 \mathrm{~h}$ (early/late-stage) or $24 \mathrm{~h}$ only (late-stage)) showed minimal differences in pathogen establishment (Fig. 5b). This was confirmed by the quantitative in vivo PET data at $24 \mathrm{~h}$ postinfection, which showed no significant difference in tracer uptake for the control group (infected but untreated mice) $(9.8 \pm 1.6 \% \mathrm{ID} / \mathrm{cc})$, mice treated with VCZ at $24 \mathrm{~h}(9.5 \pm 1.5 \% \mathrm{ID} / \mathrm{cc})$, and mice treated with $\mathrm{VCZ}$ at 3 and $24 \mathrm{~h}$ postinfection $(8.3 \pm 0.6 \% \mathrm{ID} / \mathrm{cc})$ (Fig. $5 \mathrm{c})$. However, $48 \mathrm{~h}$ after infection, qualitative differences in ${ }^{6} \mathrm{Cu}-\mathrm{hJF} 5$ accumulation in infected animals under late- or early/late-stage treatment were pronounced, with the early/late-stage treated animals showing very limited uptake of tracer in the lungs, similar to that seen in noninfected animals (Figs. $3 b$ and $5 b$ ). In contrast, there was extensive uptake of the tracer in animals receiving late-stage treatment, similar to that seen in infected, untreated animals (Figs. 3b and 5b). The quantitative in vivo PET data acquired at $48 \mathrm{~h}$, showed that animals receiving late-stage treatment had slightly increased uptake of the tracer compared to levels at $24 \mathrm{~h}$ postinfection $(11.9 \pm 2.2 \%$ $\mathrm{ID} / \mathrm{cc}$, Fig. $5 \mathrm{c}$ ), while animals receiving the early/late-stage treatment displayed significantly $(p<0.005)$ reduced accumulation
$(7.3 \pm 0.5 \% \mathrm{ID} / \mathrm{cc})$ compared to the untreated animals, and those receiving late-stage VCZ treatment. Using the volumetric approach described above, with a threshold of $10 \% \mathrm{ID} / \mathrm{cc}$, significant differences $(p<0.005)$ were already apparent at $24 \mathrm{~h}$ postinfection between animals receiving late-stage vs. early/late-stage VCZ treatment $(40 \pm 14 \%$ and $22 \pm 6 \%$, respectively, Fig. $5 \mathrm{~d})$ which was not the case when using a $15 \% \mathrm{ID} / \mathrm{cc}$ threshold. At $48 \mathrm{~h}$ postinfection, the differences in tracer uptake between the two groups was even more pronounced $(61 \pm 14 \%$ for animals receiving late-stage VCZ, and $11 \pm 4 \%$ for animals receiving early/late-stage VCZ treatment). Using a $10 \% \mathrm{ID} / \mathrm{cc}$ threshold, a significant difference between animals receiving early/late-stage treatment and the animals receiving late-stage or no treatment was found although with high volume occupation values $(11 \pm 4 \% \mathrm{ID} / \mathrm{cc}$ vs. $61 \pm 14$ and $58 \pm$ $12 \% \mathrm{ID} / \mathrm{cc}$, respectively, $p<0.0001)$. When a $15 \% \mathrm{ID} / \mathrm{cc}$ threshold was applied to the PET data as in the experimental results presented in Fig. 4d, e, no positive (infected) volume was exhibited in animals receiving early/late-stage treatment, while the animals receiving late-stage treatment displayed a $25 \pm 17 \%$ infected lung volume (Fig. 5c). Ex vivo biodistribution data obtained after perfusion of the animals (Supplementary Fig. 6) were in accordance with the in vivo findings. They showed a significantly reduced lung accumulation of ${ }^{64} \mathrm{Cu}$-hJF5 in animals receiving early/late-stage VCZ treatment compared to the late-stage animals $(12 \pm 6 \% \mathrm{ID} / \mathrm{g}$ vs. $35 \pm 14 \% \mathrm{ID} / \mathrm{g}$, respectively, $p<0.001$ ). Liver accumulation showed similar trends, with $6.9 \pm 2.6 \% \mathrm{ID} / \mathrm{g}$ for early/late-stage VCZ-treated animals, and $20 \pm 13 \% \mathrm{ID} / \mathrm{g}$ for late-stage treated animals $(p<0.05)$. While no other organs showed measurable differences in the accumulation and retention of ${ }^{64} \mathrm{Cu}-\mathrm{hJF} 5$ across the two treatment groups, animals receiving early/late-stage VCZ treatment displayed an elevated, albeit non-significant, amount of blood pool tracer. In addition, we have performed hematoxylin and eosin (H\&E) stain as well as Grocott methenamine silver stain on lung samples to observe the tissue damage and extent of the fungal invasion (Supplementary Fig. 7). The lungs of animals infected with A. fumigatus but not treated showed massive central bronchial infiltration, multiple large granulomas containing hyphae and spores, cavitations, necrosis, and destruction of the respiratory epithelium. Lungs receiving late treatment showed severe damage, with multiple and large Aspergillus granulomas containing numerous and large hyphae. Aspergillus spores were detected throughout the respiratory parenchyma. We observed large necrotic areas around the bronchi and severe destruction of the respiratory epithelium. Angioinvasion was also detected, with necrosis of the surrounding tissue. The lungs of mice receiving early treatment showed multiple small bronchio-centric inflammatory infiltrates with no significant damage of the respiratory epithelium. Few and small Aspergillus granulomas were identified, which contained exclusively spores. No hyphae were detected. The lungs of the control group (PBS instilled) showed normal histology, without pathological alterations. 

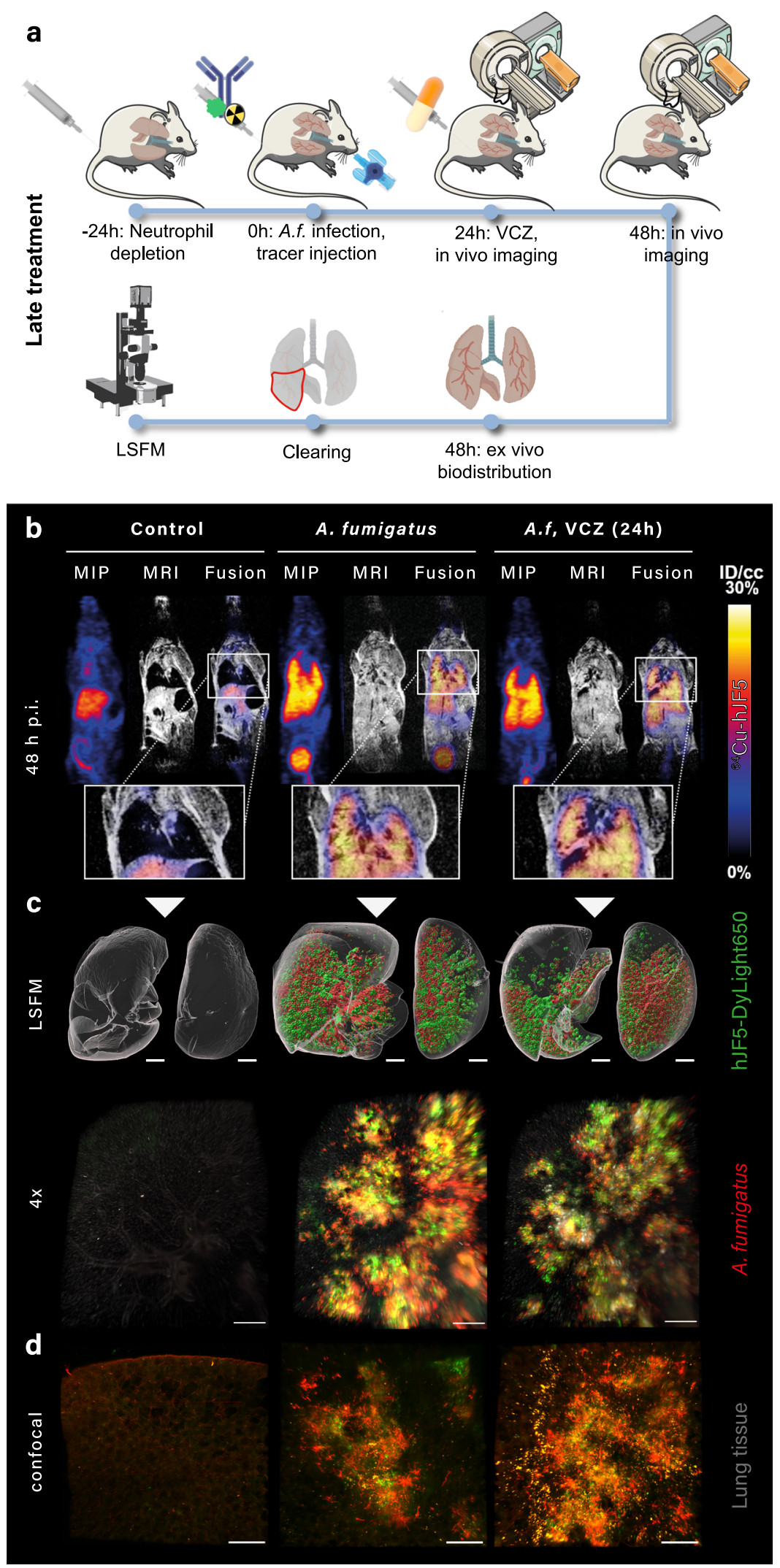

\section{Discussion}

Early and accurate diagnosis and therapy monitoring of IPA remains a significant clinical challenge due to the paucity of tests that allow specific and sensitive detection of the disease ${ }^{12}$. The recent introductions of a novel CE-marked lateral-flow device 27,28 and a CE-marked ELISA test ${ }^{29}$ that both use monoclonal antibody JF5 to detect a biomarker of Aspergillus infection have improved the speed and accuracy of IPA diagnosis, complementing the predicate galactomannan-ELISA test. Despite improved availability of biomarker tests over recent years, and significant efforts by the research community to develop noninvasive urine tests ${ }^{30,31}$, diagnosis of IPA can be delayed for days, 
Fig. 3 Impact of antifungal treatment measured with PET/MRI and LSFM using dual-labeled ${ }^{64}$ Cu-hJF5-DyLight650. a Experimental workflow depicting Aspergillus fumigatus (A.f.) infection, tracer injection, voriconazole administration (VCZ) and imaging using immunoPET/MR and LSFM. b ImmunoPET/MR images of PBS-deposited animals (Control, left, $n=2)$, A. fumigatus-infected animals without treatment (A. fumigatus, middle, $n=2)$ and animals with voriconazole treatment $24 \mathrm{~h}$ after inoculation ( $A$.f. VCZ $(24 \mathrm{~h}$ ), right, $n=3$ ). Images were acquired $48 \mathrm{~h}$ postinoculation (p.i.). MIP is a maximum intensity projection of the PET image; MRI is a T2-weighted MRI single slice MRI image; Fusion is a single slice PET image overlaid on the corresponding MRI slice. A close-up of the fusion image in the lung area is displayed below. PET data are presented as percent injected dose per cubic centimeter (\%ID/cc). c Detection of ${ }^{64} \mathrm{Cu}$-hJF5-DyLight650 with LSFM. Surface view of lungs from same animals as in $\mathbf{b}$ imaged with LSFM, and with magnified detail shown below. Lung tissue (gray), A. fumigatus (red) and ${ }^{64} \mathrm{Cu}$-hJF5-DyLight650 signal (green). Scale bar first row $=1.5 \mathrm{~mm}$, and second row $=300 \mu \mathrm{m}$. $\mathbf{d} \mathrm{High}-$ resolution confocal images of same lungs as in $\mathbf{b}$. Scale bar $=50 \mu \mathrm{m}$.

a

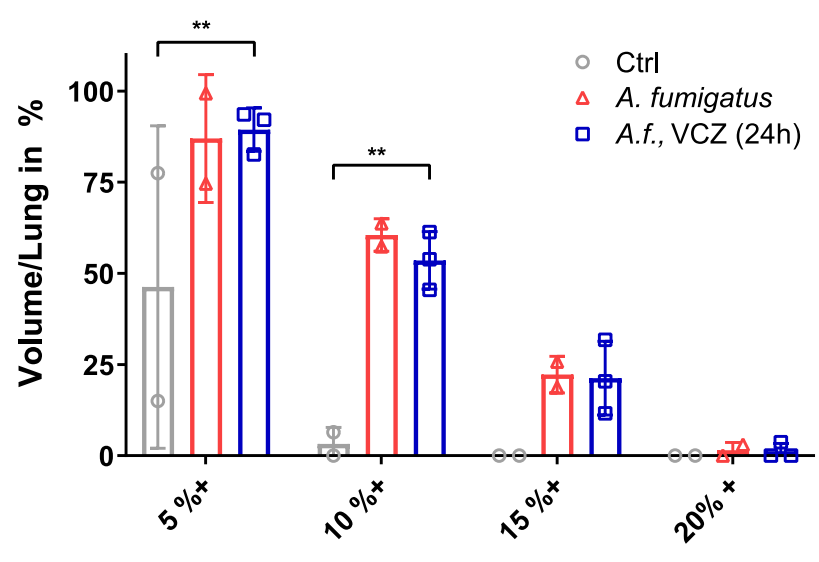

PET signal threshold in \% ID/cc
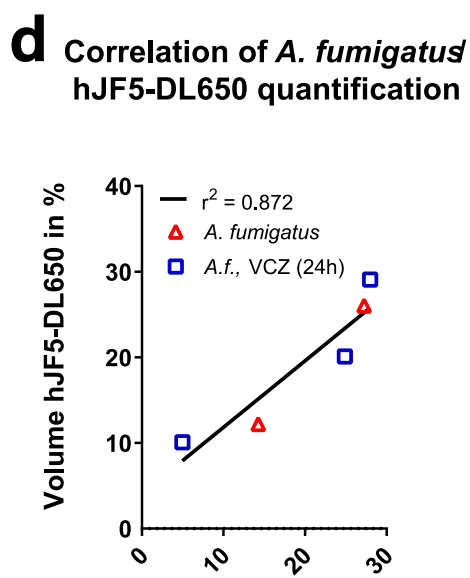

Volume A. fumigatus in \%

\section{b Infection/Lung in LSFM}

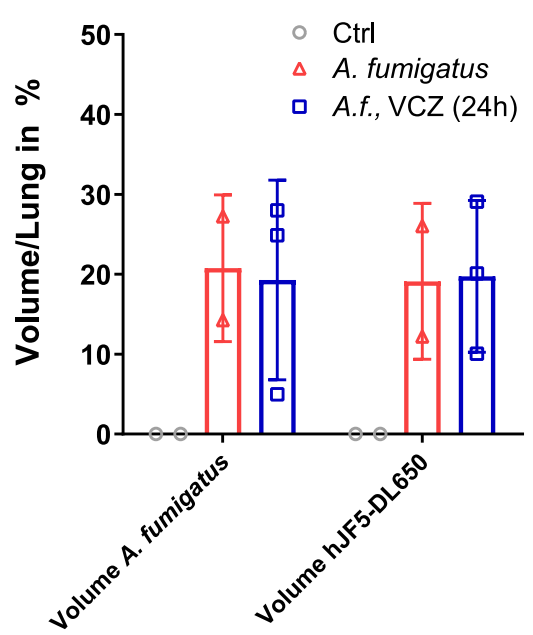

C Correlation of hJF5-DL650/
${ }^{64}$ Cu-hJF5 (15\%) quantification

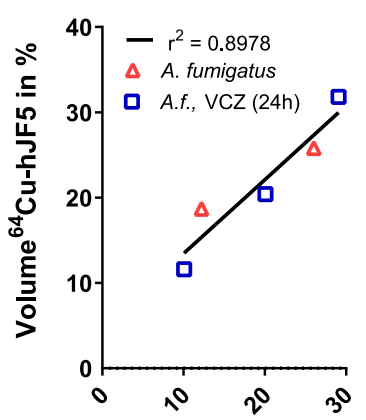

Volume hJF5-DL650 in \%

Fig. 4 Quantification and volumetric analysis of immunoPET and LSFM. a Ratio of infected lung volume as measured by immunoPET to total lung volume measured via MRI as a function of ${ }^{64} \mathrm{Cu}$-hJF5-DyLight650 accumulation threshold in percent injected dose per volume (\%ID/cc) at $48 \mathrm{~h}$ postinoculation in PBS-deposited animals (Control, left, $n=2$ ), A. fumigatus-infected animals without treatment (A. fumigatus, middle, $n=2$ ) and animals with voriconazole treatment $24 \mathrm{~h}$ after inoculation (A.f. VCZ (24h), right, $n=3$ ). 5\%: Ctrl vs. A.f., VCZ (24h), $p=0.0062 .10 \%$ : Ctrl vs. A.f., VCZ (24h), $p=0.0013$. b Volumetric ratio of $A$. fumigatus or ${ }^{64} \mathrm{Cu}$-hJF5-DyLight650 in the lung as measured by LSFM for each group. c Quantification of the co-localization of ${ }^{64} \mathrm{Cu}$-hJF5-DyLight650 and A. fumigatus ${ }^{\text {tdTomato }}$ measured by LSFM in untreated ( $A$. fumigatus, red) and treated (A.f., VCZ (24 h)). d Correlation of $A$. fumigatus invasion and ${ }^{64} \mathrm{Cu}$-hJF5-DyLight650 distribution in the lungs of $A$. fumigatus-infected animals untreated ( $A$. fumigatus, red) and VCZ treated $24 \mathrm{~h}$ after infection ( $A$.f., VCZ $\left(24 \mathrm{~h}\right.$ ) as measured by LSFM. e Correlation of ${ }^{64} \mathrm{Cu}$-hJF5-DyLight650 volumetric distribution in the lungs of $A$. fumigatus-infected animals untreated (A. fumigatus, red) and VCZ treated $24 \mathrm{~h}$ after infection ( $A$.f., VCZ ( $24 \mathrm{~h}$ ) as measured in LSFM and PET using the $15 \%$ ID/cc threshold. $\mathrm{r}^{2}$ represents Pearson's correlation coefficient. Results are plotted as individual values with average and standard deviation. $p$ values were generated using two way ANOVA with Tukey's multiple comparison test $\left({ }^{\star} p<0.05 ;{ }^{\star \star} p<0.01 ;{ }^{\star \star \star} p<0.001 ;{ }^{\star \star \star \star} p<0.0001\right)$. 

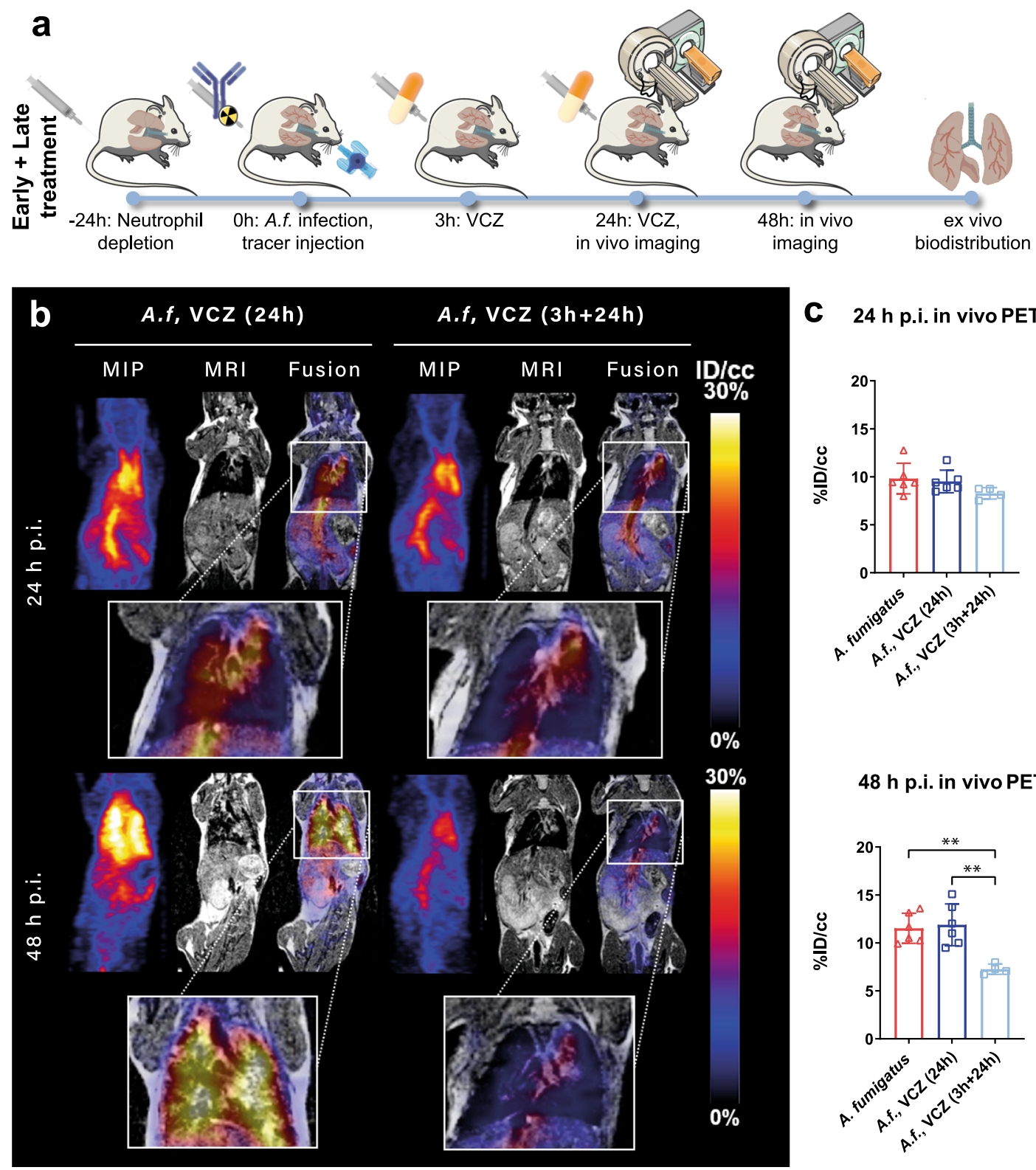

C $24 \mathrm{~h}$ p.i. in vivo PET

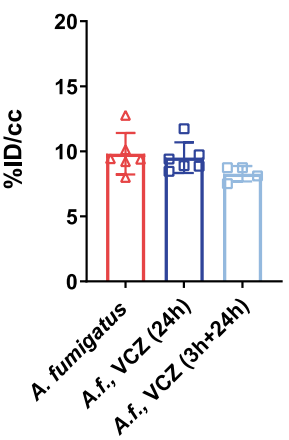

$48 \mathrm{~h}$ p.i. in vivo PET

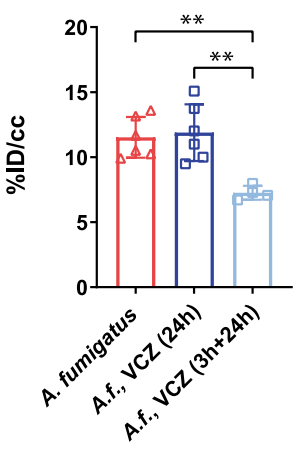

d

Infection/Lung in PET at $24 \mathrm{~h}$

Infection/Lung in PET at $48 \mathrm{~h}$

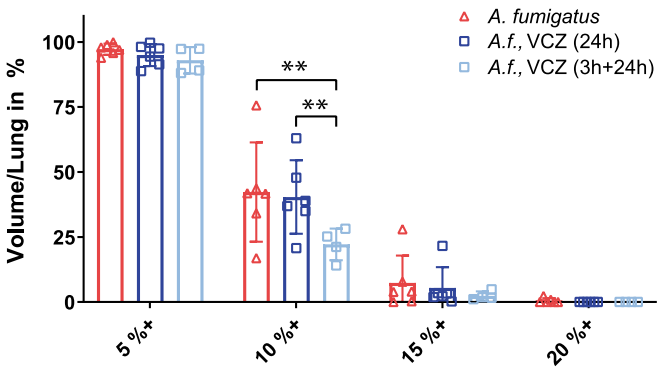

PET signal threshold in \% ID/cc

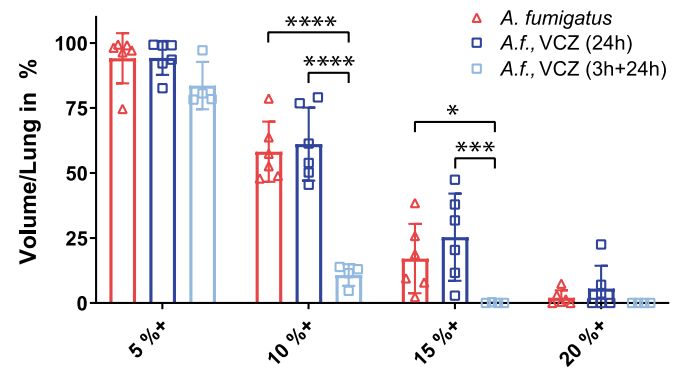

PET signal threshold in \% ID/cc weeks or even months, due to outsourcing of testing to centralized facilities. Delays in test turnaround, and the need for timely treatment of febrile patients unresponsive to antibiotics, leads to the empiric use of antifungal drugs, and limits the use of biomarker tests as tools to monitor treatment efficacies. At present, no non-invasive diagnostic procedure for IPA exists that provides sufficient specificity and sensitivity to allow for the quantitative detection of small amounts of fungus in situ, early diagnosis of Aspergillus infections in vivo, and response of the pathogen to antifungal drug treatment. To this end, the use of nuclear medicine techniques such as SPECT and PET imaging seem ideal, provided suitably specific radiotracers can be 
Fig. 5 ImmunoPET/MR evaluation of early- and late-stage voriconazole treatment on $\boldsymbol{A}$. fumigatus lung infection. a Experimental workflow depicting Aspergillus fumigatus (A.f.) infection, tracer injection, voriconazole administration (VCZ), and in vivo imaging using immunoPET/MR. b ImmunoPET/MR images of $A$. fumigatus-infected animals receiving voriconazole (VCZ) treatment initiated $24 \mathrm{~h}$ post inoculation (p.i.) $(A . f . V C Z(24 \mathrm{~h})$, left, $n=6)$, or when initiated at $3 \mathrm{~h}$ postinoculation and at $24 \mathrm{~h}$ p.i. $(A . f . V C Z(3+24 \mathrm{~h})$, right, $n=4)$. Images acquired $24 \mathrm{~h}$ p.i. are shown in the top row, while images in the bottom row show images acquired $48 \mathrm{~h}$ p.i.. MIP is a maximum intensity projection of the PET image; MRI is a T2-weighted MRI single slice MRI image; Fusion is a single slice PET image overlaid on the corresponding MRI slice. Magnifications of the fusion images of the lung are displayed below each image. c Quantification of ${ }^{64} \mathrm{Cu}$-hJF5 accumulation in the lung tissue at $24 \mathrm{~h}$ (top) and $48 \mathrm{~h}$ (bottom) p.i. of A. fumigatus in animals left untreated (A. fumigatus, red), receiving voriconazole treatment initiated $24 \mathrm{~h}$ after infection (A.f. VCZ (24 h), dark blue), or when initiated at $3 \mathrm{~h}$ postinfection and at $24 \mathrm{~h}$ postinfection (A.f. VCZ $(3+24 \mathrm{~h}$ ), light blue). $48 \mathrm{~h}$ p.i.: A. fumigatus vs. A.f., VCZ $(3 \mathrm{~h}+24 \mathrm{~h}), p=0.0046$; $A . f ., \mathrm{VCZ}(24 \mathrm{~h})$ vs. $A . f ., \mathrm{VCZ}(3 \mathrm{~h}+24 \mathrm{~h})$, $p=$ 0.0025. $\mathbf{d}$ Percentage of lung volume occupied by thresholded PET signal at $24 \mathrm{~h}$ (left) and $48 \mathrm{~h}$ (right) postinfection in the same animals. At $24 \mathrm{~h}, 10 \%$ : $\mathrm{A}$. fumigatus vs. A.f., VCZ $(3 \mathrm{~h}+24 \mathrm{~h}), p=0.0023 ;$ A.f., $\mathrm{VCZ}(24 \mathrm{~h})$ vs. A.f., $\mathrm{VCZ}(3 \mathrm{~h}+24 \mathrm{~h}), p=0.0063$. At $48 \mathrm{~h}, 10 \%$ : A. fumigatus vs. A.f., VCZ (3h +24 h), $p<0.0001$; A.f., VCZ (24 h) vs. A.f., VCZ (3h +24 h), $p<0.0001$ and 15\%: A. fumigatus vs. A.f., $\vee C Z(3 h+24 h), p=0.0321 ; A . f .$, VCZ (24 h) vs. A.f., $V C Z(3 h+24 h), p=0.0009$. Results are plotted as individual values with average and standard deviation and are expressed as percent injected dose per cubic centimeter (\%ID/cc) for PET. $p$ values were generated using one way ANOVA (c) or two way ANOVA (d) with Tukey's multiple comparison test $\left({ }^{\star} p<0.05 ;{ }^{\star \star} p<0.01 ;{ }^{\star \star \star} p<0.001 ;{ }^{\star \star \star \star} p<0.0001\right)$.

developed that enable high affinity and selectivity for IPA. While recent studies suggest that $\left[{ }^{18} \mathrm{~F}\right]$ FDG-PET/CT might help discriminate between active and residual fungal lesions to support decisions for safely stopping antifungal treatments ${ }^{32}$, we have shown that the metabolic tracer $\left[{ }^{18} \mathrm{~F}\right] \mathrm{FDG}$ is not specific for IPA, and is unable to discriminate between Aspergillus infections, bacterial infections, and inflammatory processes of the lung ${ }^{19}$. Alternative strategies for imaging IPA are therefore needed. The three main alternatives investigated to date are (1) the use of radiolabeled antifungal drugs, which show poor ability to detect A. fumigatus infections, and to differentiate these from infections by other fungal pathogens 33,34 ; (2) the use of radiolabeled siderophores ${ }^{30,35,36}$ which, while demonstrating significant potential in animals models of disease, may lack sufficient specificity, and show reduced sensitivity in patients with iron overload (typically those at high risk of developing IPA including heavily transfused AML patients, neutropenic patients, liver and allogeneic HSCT recipients, and those receiving chemotherapy); ${ }^{13}$ (3) the use of an Aspergillus-specific mAb, JF5, which allows highly specific and sensitive detection of $A$. fumigatus lung infections in vivo ${ }^{13,19,20}$, and which has been humanized, via CDR grafting into a human IgG1 framework, for use as a diagnostic tracer in humans ${ }^{20}$. In parallel, optical detection of $A$. fumigatus has been shown in a fungal keratitis model using fluorescent liposomes, achieving rapid and sensitive diagnosis, although with an imaging depth too limited for lung imaging 37 .

In this study, we extend our initial work to examine whether and how the hJF5 antibody can be used for early diagnosis and therapy monitoring of IPA. By coupling the antibody to the radionuclide ${ }^{64} \mathrm{Cu}$ and the fluorophore DyLight 650 , we have gained insight into the progression of $A$. fumigatus lung infections in vivo, and the response of infections to early- and latestage treatment with the antifungal drug VCZ. Using light sheet fluorescence microscopy (LSFM), with a mesoscopic sample size similar to whole animal immunoPET, we were able to quantify, in $3 \mathrm{D}$, uptake of the antibody tracer and development of a genetically modified strain of the pathogen (A. fumigatus $\left.{ }^{\text {tdTomato }}\right)$, in parallel, in situ. We were therefore able to visualize growth of the fungus under different conditions in the intact lung both in vivo and ex vivo, including the role of the neutropenic environment on pathogen development, and the timing of therapy on disease progression. This demonstrates the utility of immunoPET as a nondestructive and noninvasive disease-monitoring tool to observe the in vivo response of A. fumigatus to antifungal drug treatment. Previous attempts to study the consequences of antifungal therapy on disease progression have relied on destructive, invasive, or semi-invasive procedures including histopathological examination of diseased lung tissues ${ }^{22,38}$, culture of invasive BALf samples for estimates of fungal load ${ }^{7}$, and circulating biomarkers as proxies of disease burden $22,38-40$. It is important to mention that, while our efforts and model were based on the Aspergillus fumigatus species, the JF5 antibody recognizes and binds to all Aspergillus species and thus still holds potential in parts of the world where $A$. flavus or A. terreus can be the most common cause of IPA $^{18}$. In addition, we have focused on the lung presentation of the disease. While rare, cerebral aspergillosis can also occur, especially in patients treated with ibrutinib. Here, our chosen antibody-based approach might be limited as it would require breakdown of the blood brain barrier $(\mathrm{BBB})$ to allow for radiolabeled antibody accumulation at the site of infection. However, A. fumigatus metabolites such as gliotoxins ${ }^{41}$ have been shown to impair BBB integrity.

Neutrophils play critical roles in host defence to fungal infections ${ }^{42}$. In our neutropenic mouse model of IPA, neutrophil deficiency, more specifically loss of $\mathrm{Gr}-1^{+}$cells, resulted in infection of the entire lung volume within a matter of hours of challenge with spore inoculum, whereas, in the presence of neutrophils, the pathogen was rapidly controlled. This demonstrates further the critical role of $\mathrm{Gr}-1^{+}$neutrophils in preventing infectious pathogens ${ }^{43}$, and provides an additional, and highly effective, means of depleting neutrophils, to those used by Amich and co-workers in LSFM of $A$. fumigatus lung infections ${ }^{44}$. Surprisingly, in healthy animals, neutrophil-mediated control of lung infection took longer than expected, as invasion was apparent in LSFM $6 \mathrm{~h}$ after initial deposition of A. fumigatus spores. However, the spore concentrations used in our investigations were superficially high in order to obtain a complete and rapid invasion of the lung, and were several orders of magnitude higher than the daily estimated inhalation of $A$. fumigatus spores in real-life ${ }^{45}$. Nevertheless, the timing of the different stages of lung infection (conidial germination, hyphal proliferation, and invasive of the contiguous lung) are similar to those reported in other neutropenic models of IPA 22,38

In order to determine the effectiveness of our molecular imaging approach as a means of monitoring the in vivo response of invasive lung infection to antifungal treatment, we administered the azole drug VCZ at early $(3 \mathrm{~h})$ and late $(24 \mathrm{~h})$ time points postinoculation, and then quantified spatio-temporal changes in pathogen load using immunoPET. In PET imaging, no effect of the drug was found on the distribution and accumulation of ${ }^{64} \mathrm{Cu}-\mathrm{hJF} 5$ in the lungs or other organs in infected animals, when administered $24 \mathrm{~h}$ postinoculation. Even at $48 \mathrm{~h}$ postinoculation, $24 \mathrm{~h}$ after the drug was administered, no control of the disease was evident. A possible explanation for this might be the biological activity of VCZ, "holding" A. fumigatus 46 infections by a fungistatic effect until the immune system can take over. While 
the effect of VCZ in limited time frames is, to the best of our knowledge, undocumented in vivo, its initial activity in vitro is likely to be fungistatic, with fungicidal activity following $24-48 \mathrm{~h}$ earliest after initial fungal exposure ${ }^{47-49}$. Data from LSFM of the lungs confirmed the immunoPET data, and similarly showed a lack of reduction in IPA following late-stage administration of VCZ. Our data showed that, in order to be effective, VCZ must be administered as early as possible during the infection process in the setting of neutropenia, with early/late-stage treatment providing complete remission of the disease. Our findings are consistent with previous studies which have shown that early intervention with antifungal drugs is critical to control of IPA ${ }^{38}$. Our work has also demonstrated the power of JF5-guided imaging as a means of tracking IPA in vivo during the course of antifungal drug treatment. As a highly sensitive and diseasespecific tracer, and one that detects active growth of $A$. fumigatus ${ }^{13,18-20},{ }^{64} \mathrm{Cu}$-hJF5 represents a manifest improvement over $\left.{ }^{18} \mathrm{~F}\right]$ FDG-PET/CT ${ }^{32}$ as a means of discriminating between invasive growth and resolution of infection. Accumulation of the hJF5 tracer was similar in both treated and non-treated animals, and correlated exclusively with the distribution of the pathogen. This demonstrates the absence of false positive accumulation (hJF5 accumulating in A. fumigatus-absent spaces) and false negativity (hJF5 not accumulating in A. fumigatusrich spaces). In addition, the ex vivo results showed a discrepancy between radiotracer uptake in the spleen of animals infected with A. fumigatus compared to animals with a sham inoculation. This might point toward dissemination of the pathogen to the spleen, which has been reported several times in humans ${ }^{50,51}$. However, it might alternatively demonstrate accretion of soluble antigen released into the bloodstream during invasion of the lung.

While the extended circulation time of full-length antibodies (days to weeks) and their high bio-availability are a significant advantage in many instances, these features do present an important limitation for immunoPET in vivo, which is a high blood pool of tracer. In our system, this typically manifested as blood quantities of $>20 \% \mathrm{ID} / \mathrm{g}$ in ex vivo biodistribution assays and, more importantly, in vivo accumulation of ${ }^{64} \mathrm{Cu}$-hJF5 in the highly perfused lungs of noninfected control animals. Biodistribution studies showed a difference in blood circulation values between infected (treated and non-treated) and control animals, which we attribute to circulating antigen. We believe this PET signal still originates from immunoreactive radiolabeled antibody, as we have shown in previous studies the stability of ${ }^{64} \mathrm{Cu}$ chelation to NODAGA-hJF5 as well as the conjugation between NODAGA and hJF5 ${ }^{20}$. Since it is not possible to distinguish blood vessels from lung tissue in immunoPET/MR, this translates to a background signal that reduces the contrast between healthy and diseased tissues in in vivo PET/MR. To mitigate this, we conducted ex vivo measurements after thorough perfusion of the animals with PBS in order to remove any unbound circulating ${ }^{64} \mathrm{Cu}$-hJF5 thereby allowing the distinction between circulating radiotracer and bound immunoglobulin. The benefits of this approach were clearly evident in LSFM, where control animals showed no fluorescence signal at all due to ${ }^{64} \mathrm{Cu}-\mathrm{hJF5}$ DyLight650. Furthermore, the accumulation of ${ }^{64} \mathrm{Cu}-\mathrm{hJF} 5$ determined in ex vivo biodistribution assays was $\sim 10$-fold lower in control animals compared to infected animals, and an approximate 2 -fold difference was achieved in in vivo PET at the same time point. While this is a sizeable difference, it is very likely a feature unique to the murine model of IPA since the lungs are fully invaded by small nodules of fungus, which is apparent in the LSFM images. This results in a dispersed mass at the resolution of the PET system $(\sim 1 \mathrm{~mm})$. This might lead to a partial volume effect, ultimately lowering the estimated PET accumulation values. In the case of a naturally infected human, IPA generally presents as several compact nodules of a few centimeters against a healthy tissue background ${ }^{52}$. Given the organ and nodule size in relation to the conserved PET resolution, the contrast ratio between positive nodules and healthy lung tissue should increase considerably, and prove sufficiently different, for human disease diagnosis and therapy monitoring. A possible means of reducing the blood pool retention of the radiotracer, is to shorten the circulation time of the targeting vector through chemical manipulation. Antibody fragments are a classical approach, as smaller fragments often conserve the affinity to the target of interest but with a more limited circulation time, with typical half-life times of $10 \mathrm{~h}$ for $\mathrm{F}\left(\mathrm{ab}^{\prime}\right)_{2}$ fragments or even less than $1 \mathrm{~h}$ for nanobodies ${ }^{53}$. Such strategies are currently being investigated by our group to achieve a higher contrast ratio between diseased and healthy tissue at a faster rate than currently achievable with a full-length radiolabeled antibody.

Notwithstanding this, and in order to achieve a higher contrast ratio in vivo as well as better nodule delineation and disease staging, we developed a volumetric thresholding method applicable to both LSFM and PET images. This allowed the values acquired in vivo and ex vivo to be correlated, and provided a more robust mechanism for disease detection. To achieve this, a delineation of the organ was performed using a reference anatomical image (MRI or autofluorescence for immunoPET and LSFM, respectively), and the volume occupied by the specific signal above a defined threshold value calculated. Initially, we used a threshold value similar to that of the average background levels $(5 \% \mathrm{ID} / \mathrm{cc})$, which yielded too much noise and variance to allow conclusive results. While using a $10 \% \mathrm{ID} / \mathrm{cc}$ threshold provided more meaningful results, the volumes occupied by the pathogen were largely over-estimated when compared to the results obtained by LSFM. This could be due to the high perfusion of the lung (and blood containing $\sim 20 \% \mathrm{ID} / \mathrm{g}$ ), giving false positive signals indistinguishable from true nodule signals achievable at the resolution of small animal PET. By increasing the threshold to $15 \% \mathrm{ID} / \mathrm{cc}$, representing the maximal PET signal in healthy animal lungs, we were able to obtain a clear delineation of the diseased regions of the lungs, often matching with MRI T2 contrast, with values similar to the LSFM values obtained ex vivo. Furthermore, we achieved a significantly higher ratio between diseased and control lungs in vivo than previously achieved using classical PET quantification approaches. This effectively solved the main immunoPET issue of high blood pool retention of $\mathrm{mAb}$ tracer.

In conclusion, we have shown that hJF5 can be used successfully in vivo as a diagnostic and therapy monitoring tool for IPA, accurately tracking changes in pathogen load in the neutropenic lung during disease progression and in response to antifungal drug treatment. It is important to note that while this murine model of IPA is an accurate representation of the clinical aggressiveness of the pathogen in the context of immuno-suppression, clinical presentation of IPA is typically as discrete nodules rather than diffuse invasion of the contiguous lung. In this event, the volumetric threshold procedure developed here might prove useful for clinical diagnosis of the disease using antibody-guided molecular imaging. We foresee that the main applications of this immunoPET approach could be firstly to rulein or rule-out Aspergillus lung infection, and secondly to aid identification of treatment end-point, improving clinical decisions regarding duration of the antifungal therapy.

\section{Methods}

Preparation of labeled hJF5. For conjugation of hJF5 to NODAGA, purified hJF5 antibody was first incubated with EDTA $(0.5 \mathrm{mM})$ for $45 \mathrm{~min}$ at room temperature under gentle stirring to remove metal contamination. Buffer exchange and EDTA excess removal was then performed using trace-metal basis $0.1 \mathrm{M}$ boric acid 
(Sigma-Aldrich, Switzerland), pH 9.1, equilibrated with PD-10 (GE Healthcare, Switzerland). Amicon Ultra-15 centrifugal filter units with a $10 \mathrm{kDa}$ molecular weight cut-off (Merck Millipore, Switzerland) were then used for antibody collection. p-NCS-Bz-NODAGA (Chematech, France) was dissolved in DMSO at $33.3 \mathrm{mM}$ and applied at a 20 -fold molecular excess to the antibody solution $(33.3 \mu \mathrm{M})$ and the reaction took place for $16 \mathrm{~h}$ at $4{ }^{\circ} \mathrm{C}$. After purification, performed with a PD-10 column, the antibody was equilibrated in Chelex-treated PBS, pH 7.4 (Bio-Rad, Switzerland). Filtration using Amicon Ultra-15 centrifugal filter units (10 kDa cut off) and two further purification steps using Chelex-treated PBS (pH 7.4) followed ${ }^{19}$

For dual-labeled hJF5, the chelator-conjugated antibody was further conjugated with a 10 -fold molar excess of DyLight 650 NHS ester (Thermo Scientific, Rockford, IL, USA) according to the manufacturer's instructions. The conjugate was purified using a PD-10 column equilibrated with phosphate-buffered saline. The antibody was radiolabeled by incubation with $0.5 \mathrm{MBq}{ }^{64} \mathrm{Cu}$ per $\mu$ g for $60 \mathrm{~min}$ at $42^{\circ} \mathrm{C}$

${ }^{64} \mathrm{Cu}$ was produced in house by $12.5 \mathrm{MeV}$ proton irradiation of enriched ${ }^{64} \mathrm{Ni}$ metal electroplated on a platinum/iridium disc via the ${ }^{64} \mathrm{Ni}(\mathrm{p}, \mathrm{n}){ }^{64} \mathrm{Cu}$ nuclear reaction, and purified after acidic dissolution by anion exchange chromatography. Antibodies coupled to NODAGA were then radiolabeled using $0.5 \mathrm{MBq}$ of acetatebuffered ${ }^{64} \mathrm{CuCl}_{2}$ per $\mu \mathrm{g}$ of antibody.

Quality control was performed using thin layer chromatography (Polygram SIL G/UV254, Macherey-Nagel; mobile phase: $0.1 \mathrm{M}$ sodium citrate, $\mathrm{pH}$ 5.0) and HPSEC (Phenomenex BioSep SEC-s3000 $300 \times 4.6 \mathrm{~mm}, 1.5 \mathrm{ml} / \mathrm{min}$ saline sodium citrate). Reactivity of antibody conjugates was confirmed by ELISA with purified antigen as described previously ${ }^{20}$.

\section{Aspergillus fumigatus strains and voriconazole susceptibility testing. The} Aspergillus fumigatus wild-type strain ATCC 46645 and strain A. fumigatus ${ }^{\text {tdTomato }}$ (strain ATCC 46645 with insertion of the tdTomato gene via pSK379-Tomato plasmid in the His2 sequence ${ }^{54}$ ) were grown on defined minimal medium for three days at $37^{\circ} \mathrm{C}$ before resting conidia were harvested from sporulating mycelium with tap water $+0.1 \%$ Tween-20. The growth medium of the A. fumigatus $\mathrm{dT}$ Tomato strain was supplemented with $0.1 \mu \mathrm{g} / \mathrm{ml}$ pyrithiamine hydrobromide (SigmaAldrich, St. Louis, Missouri, USA) as selection marker. Spore suspensions were filtered through a series of cell strainers with decreasing mesh size (100, 30, and 10 $\mu \mathrm{m})$ before spore concentration was assessed using an automated cell counting system (Cellometer Auto T4; Nexcelcom, Manchester, UK). After adjusting the spore concentration to $3 \times 10^{5}$ spores $/ \mathrm{ml}$ with deionized water, $100 \mu \mathrm{l}$ of these suspensions were used per well to inoculate the EUCAST 96-well flat-bottom testing plates (TPP, Trasadingen, Switzerland). Previously, these standard cell culture plates were filled with $100 \mu$ d double-concentrated EUCAST medium (RPMI 1640 (with glutamine and a pH indicator but without bicarbonate) $+[4 \%]$ (w/v) Glucose $+[0.33 \mathrm{~mol} / \mathrm{l}] 3$-(N-morpholino) propanesulfonic acid (MOPS)) per well with which double-concentrated dilution series of voriconazole (Vfend; Pfizer, New York City, USA; reconstituted with sterile, deionized water) were generated $(32,16,8,4,2,1,0.5,0.25,0.125,0.06 \mu \mathrm{g} / \mathrm{ml})$. By adding $100 \mu \mathrm{l}$ of the spore suspensions to the wells of the 96-well plates the total spore number per well equated to $3 \times 10^{4}$, the EUCAST medium was diluted to become $1 \times$ concentrated (RPMI $1640+[2 \%]$ Glucose $+[0.165 \mathrm{~mol} / \mathrm{l}] \mathrm{MOPS}$ ), and the final voriconazole concentrations were adjusted to $16,8,4,2,1,0.5,0.25,0.125,0.06,0.03 \mu \mathrm{g} / \mathrm{ml}$. For each strain, two individual spore preparations were used (=biological replicates) from which three separate voriconazole dilution series were inoculated (=technical replicates). The inoculated plates were then incubated for $48 \mathrm{~h}$ at $37^{\circ} \mathrm{C}$ before the MIC values were determined. For visual MIC determination, all inoculated testing rows were investigated with a stereo microscope (S8 APO; Leica Microsystems, Wetzlar, Germany) for the first well of the row in which no fungal growth was detectable. Subsequently, for assessing the MIC values spectrophotometrically, the absorbance at $490 \mathrm{~nm}$ of all inoculated wells of the 96-well plates was measured (iMark; Biorad, Hercules, California, USA). As blank control, fresh $1 \times$ concentrated EUCAST medium was used.

Mouse strain. For all experiments, C57BL/6JOlaHsd mice were used. Eight to tenweek-old female mice were purchased from Envigo (Huntingdon, Cambridgeshire, $\mathrm{UK})$, and kept in a standardized and sterile environment $\left(20 \pm 1^{\circ} \mathrm{C}\right.$ room temperature, $50 \pm 10 \%$ relative humidity, $12 \mathrm{~h}$ light-dark cycle), with food and water provided ad libitum. All experiments were undertaken in accordance to the animal welfare act and the EU directive 2010/63/EU, the German animal welfare act and after review and approval by the responsible local ethical committees and authorities (Regierungspräsidium Tübingen and Essen, Germany).

A. fumigatus infection and treatment. Mice were rendered neutropenic by an intraperitoneal (i.p.) injection of 100-200 $\mu \mathrm{g}$ anti-Ly6G/anti-Ly6c antibody (BioXCell, West Lebanon, USA) diluted to $1 \mathrm{mg} / \mathrm{ml}$ in PBS (DPBS, Gibco Life technologies, Carlsbad, CA, USA) $18-24 \mathrm{~h}$ prior to fungal infection or injected by an equivalent amount of isotype antibody for the isotype-depleted group. For the infection procedure, mice were first anaesthetized with $100 \mu \mathrm{l}$ of Ketamin/Xylazin or Rompun solution i.p. (Ketamin: $80-100 \mathrm{mg} / \mathrm{kg}$. Ratiopharm GmbH, Ulm; Rompun: $15 \mathrm{mg} / \mathrm{kg}$. Bayer HeathCare, Leverkusen or Xylazin 2\%: $10 \mathrm{mg} / \mathrm{kg}$. WDT,
Garbsen), before intubation using a 22-gauge in-dwelling venous catheter (Vasofix Braunüle, B. Braun AG, Melsungen, Germany). Correct intubation in the trachea was confirmed by ventilation, before deposition of a suspension of $4-5 \times 10^{6}$ spores of A. fumigatus in 50-100 $\mu$ l PBS (MiniVent, Hugo Sachs, March-Hugstetten, Germany). Either wild-type A. fumigatus or A. fumigatus ${ }^{\text {tdTomato }}$ were used depending on the experiment. Complete distribution in the lungs was obtained by artificial ventilation (250-300 $\mu \mathrm{l} / \mathrm{breath}$ at $200-250 \mathrm{breaths} / \mathrm{min}$ for $1 \mathrm{~min}$ using a small animal respirator). Control animals received an equal volume of the PBS carrier only.

For immunoPET/MRI experiments, mice were then randomly split into groups, and received the following treatments: (1) no antifungal treatment (A. fumigatus only group); (2) a single i.v. injection of $6 \mathrm{mg} / \mathrm{kg}$ of VCZ (Hexal, Holzkirchen, Germany) $24 \mathrm{~h}$ after infection (late-stage VCZ treatment); (3) an i.v. injection of 6 $\mathrm{mg} / \mathrm{kg}$ of VCZ $3 \mathrm{~h}$ after infection, followed by a second injection of $4 \mathrm{mg} / \mathrm{kg}$ of VCZ $24 \mathrm{~h}$ after infection (early/late-stage VCZ treatment).

Immediately after infection, mice were separated by experimental groups to avoid cross contamination, in litters of maximum 6 littermates. Thus, no blinding of the investigator was possible.

In vivo immunoPET/MR imaging. Directly after intratracheal infection of the animals, $\sim 12.5 \mathrm{MBq}$ of radiolabeled hJF5, corresponding to $\sim 25 \mu \mathrm{g}$ of protein $\left({ }^{64} \mathrm{Cu}-\mathrm{hJF5}\right.$ injected $3.04 \mathrm{mg} / \mathrm{ml},{ }^{64} \mathrm{Cu}-\mathrm{hJF} 5$-DyLight650 at $\left.1.25 \mathrm{mg} / \mathrm{ml}\right)$ at, was injected i.v. In vivo imaging took place 24 and $48 \mathrm{~h}$ after initial infection of the animals using a small animal PET system (Inveon ${ }^{\mathrm{m}}$, Siemens Healthcare $\mathrm{GmbH}$, Erlangen, Germany), using the Inveon Acquisition Workplace (IAW) v2.1.272 software (Siemens Preclinical Solutions, Knoxville, TN, USA) thoroughly calibrated ${ }^{55}$ and undergoing daily quality and linearity response controls, ensuring reproducibility of measurements, and a small animal MRI using the Paravision v6 software (7 T Bruker Biospin GmbH, Billerica, MA, USA). Before each imaging session, the animals were weighed and their appearance was checked for physical or behavioral abnormalities. During the scans, the animals were anaesthetized with $0.8-2.5 \%$ isoflurane in $100 \%$ oxygen (depending on their health and fitness). Anesthesia was monitored by measuring the breathing frequency. Mice were positioned into a PET and MRI transparent animal holder alongside two capillaries containing a minimal amount of radioactivity for coregistration of PET and MRI imaging data. For acquisition, a $600 \mathrm{~s}$ static PET acquisition sequence was used followed by two MRI sequences (T2_TurboRARE (TR/TE $600 \mathrm{~ms} / 35 \mathrm{~ms}$, size $256 \times 128 \times 92 \mathrm{~mm}$, slice thickness $23 \mathrm{~mm}$, field of view (FOV) $64 \times 32 \times 12 \mathrm{~mm}$, and resolution $0.25 \times 0.25 \times 0.25)$ and T1-FLASH (TR/TE $12 / 3 \mathrm{~ms}$, FA $8.9^{\circ}$, size $160 \times 80 \times 60 \mathrm{~mm}$, slice thickness $18.868 \mathrm{~mm}$, FOV $50 \times 25 \times 18.868 \mathrm{~mm}$, and resolution $0.314 \times 0.314 \times 0.314)$ ). PET image reconstruction was performed using a $3 \mathrm{D}$ ordered subset expectation maximization (3D-OSEM) with no attenuation correction in IAW and co-registered with MRI images using in Inveon Research Workplace software v4.2 (Siemens Preclinical Solutions, Knoxville, TN, USA). Region of interest (ROI) was drawn onto the lungs using the MR Images for guidance, and the PET data were used to extract percent injected dose per cubic centimeter $(\mathrm{cc})[\% \mathrm{ID} / \mathrm{cc}]$ in the lung. Lung volumes were measured using the ROI as delineated in the MRI, or measured based on the PET data after thresholding the initial lung ROI at a defined \%ID/cc value.

Ex vivo analysis. After the last in vivo imaging scans, retro-bulbar blood samples were obtained from the animals before $\mathrm{CO}_{2}$ sacrifice. Shortly thereafter, all animals were perfused through the left ventricle with $0.4 \%$ of PFA (Sigma-Aldrich, Darmstadt, Germany) in $20 \mathrm{ml}$ of $4{ }^{\circ} \mathrm{C}$ PBS. Afterwards, organs were removed and radioactivity was quantified with an aliquot of the injected radiotracer in a $\gamma$-counter (Wallac 2480 WIZARD 3", PerkinElmer, Waltham, MA, USA) using an energy window between 350 and $650 \mathrm{keV}$. The results are expressed as \% injected dose per gram $(\% \mathrm{ID} / \mathrm{g})$ of tissue.

CUBIC clearing and 3D fluorescence microscopy. Organs prepared for 3D fluorescence microscopy were cleared with the CUBIC protocol as previously described $^{56}$. All incubation steps were performed in the dark. Fixated lungs were transferred to $0.01 \% \mathrm{NaN}_{3} / \mathrm{PBS}$ (Sigma-Aldrich, Darmstadt, Germany) overnight at RT with shaking. After an additional washing step with $0.01 \% \mathrm{NaN}_{3} / \mathrm{PBS}$, the lungs were incubated in $50 \%$ CUBIC reagent- $1 / \mathrm{dH}_{2} \mathrm{O}$ and $3 \mathrm{~h}$ at RT with shaking Reagent-1 was prepared according to the published protocol ${ }^{56}$ and was comprised of 25\% Urea (Sigma-Aldrich, Darmstadt, Germany), 25\% Quadrol (Sigma-Aldrich, Darmstadt, Germany) and 15\% Triton X-100 (Sigma-Aldrich, Darmstadt, Germany) in $\mathrm{dH} 2 \mathrm{O}$. On the next day, the samples were transferred in $100 \%$ reagent- 1 over night at RT with continuous shaking, followed by replacement of the solvent by fresh reagent- 1 on the next day for an additional $24 \mathrm{~h}$ period incubation. Following the incubation period, $3 \times 2 \mathrm{~h}$ washing cycles at RT with $0.01 \% \mathrm{NaN} 3 / \mathrm{PBS}$ were performed with shaking and the lungs immediately immersed in 50\% CUBIC reagent-1/PBS over night at RT. Reagent-2 was prepared following the established protocol $^{56}$ with $25 \%$ urea, $50 \%$ sucrose (Sigma-Aldrich), and $10 \%$ triethanolamine (Sigma-Aldrich) in $\mathrm{dH}_{2} \mathrm{O}$. Final clearing was performed in pure reagent-2 overnight at RT with gentle shaking, followed by a last change of reagent- 2 for at least $24 \mathrm{~h}$ at RT, with shaking. Before image acquisition, samples were preincubated in a 
mixture of 50\% silicon oil (Sigma-Aldrich) and 50\% mineral oil (Sigma-Aldrich) 30 min, before imaging in the same oil composition.

All samples were imaged using an Ultramicroscope II and ImSpector software (LaVision BioTec, Bielefeld, Germany). The microscope is based on a MVX10 zoom body (Olympus, Hamburg, Deutschland) with a $\times 2$ dipping objective, and equipped with a Neo sCMOS camera (Andor, Oxford instruments, Tubney Woods, Abingdon, UK). Samples were imaged along the coronal axis in a commercially available sample holder and cuvette (LaVision BioTec), with a total magnification of $\times 1.26$ or $\times 4$ and a $z$ spacing of 10 or $5 \mu \mathrm{m}$ between optical planes. A FOV with $2560 \times 2160$ pixel and a diagonal of 17.6 or $5.5 \mathrm{~mm}$ resulted in a $x / y$ pixel size of 5.16 or $1.62 \mu \mathrm{m}$. For image acquisition, samples were excited with light sheets of the wavelengths 488, 561, and $639 \mathrm{~nm}$. The longest wavelength was imaged first and shortest last to avoid photobleaching during imaging. A bandpass emission filter with a center wavelength of $525 \mathrm{~nm}$ and a bandpass width of $50 \mathrm{~nm}(525 / 50)$ was used for the detection of autofluorescence, 585/40 for the detection of the tdTomato signal of A. fumigatus and 680/30 for the DyLight650 signal of the hJF5 antibody.

Multiphoton images of fungal development were generated from the right inferior lobes using the Leica TCS SP8 MP and Leica Application Suite (LAS X) software (Version 3.1.5.16308; Leica Microsystems GmbH, Wetzlar, Germany). Zstacks of $150 \mu \mathrm{m}$ with a spacing of $0.5 \mu \mathrm{m}$ were acquired using the $2.8 \mathrm{~W}$ Chameleon Vision II Titan-Sapphire laser with an excitation wavelength of 960 $\mathrm{nm}$. A $\times 25$ objective (Leica Microsystems $\mathrm{GmbH}$ ) with a $\times 1.5$ zoom and a scan speed of $100 \mathrm{~Hz}$ resulted in a FOV with the size of $295.24 \mu \mathrm{m} \times 295.24 \mu \mathrm{m}$ in $1024 \times 1024$ pixels, and a $x / y$ pixel size of $0.289 \mu \mathrm{m}$. Autofluorescence was detected with a photomultiplier tube (PMT) bandpass emission filter 525/50, and tdTomato signal with a hybrid detector (HyD) 585/40.

Confocal images of the whole lungs, first imaged using PET/MRI, were acquired using the Leica TCS SP8 gSTED equipped with a $1.5 \mathrm{~mW}$ tuneable white light laser and LAS X software (Version 3.5.5.19976; Leica Microsystems GmbH). With a $\times 20$ objective (Leica Microsystems $\mathrm{GmbH}$ ), excitation wavelengths of $488 \mathrm{~nm}(52 \%)$, $554 \mathrm{~nm}(63 \%), 652 \mathrm{~nm}(63 \%)$ and a scan speed of $400 \mathrm{~Hz}$, z-stacks of around 200 $\mu \mathrm{m}$ with a spacing of $1 \mu \mathrm{m}$ were acquired. A FOV with the size of $581.25 \mu \mathrm{m} \times$ $581.25 \mu \mathrm{m}(2048 \times 20,148$ pixels $)$ results in a $x / y$ pixel size of $0.284 \mu \mathrm{m}$. Autofluorescence was detected with a tuneable PMT of $499-525 \mathrm{~nm}$, tdTomato signal with a tuneable HyD of 561-626 nm, and DyLight650 with an HyD of $670-718 \mathrm{~nm}$.

Image processing. Recorded $z$-stacks ( 16 bit OME.TIF) were converted into Imaris files (.ims (Bitplane AG, Oxford instruments, Zurich, Switzerland)) using the ImarisFileConverterx64 (Version 9.4.X and 9.5.X). The 3D reconstruction of the surfaces, and the subsequent analyses, were performed with Imaris (Version 9.5.X, Bitplane). The displayed surface of A. fumigatus ${ }^{\text {tdTomato }}$ signal was built using a threshold from 1000 to 3969.38 counts, and the displayed surface of hJF5DyLight650 signal with 500 to 1117.71 counts. For the co-localization of both signals, we reconstructed a new surface from the A. fumigatus surface with the threshold values from the hJF5 surface reconstruction and calculated the overlap.

Histology. After sacrifice, animals were perfused with $20 \mathrm{ml}$ PBS at $4{ }^{\circ} \mathrm{C}$ before organ removal. Whole lungs were fixed in $4 \%$ formalin and paraffin embedded. Tissue sections of 3-5 $\mu \mathrm{m}$ thick were prepared and stained with H\&E and Grocott to demonstrate fungal structures. Appropriate positive and negative controls were used to confirm the adequacy of the staining. Photomicrographic images were acquired with an Axioskop 2 plus Zeiss microscope equipped with a Jenoptik (Laser Optik System, Jena, Germany) ProgRes C10 plus camera and software. Final image preparation was performed with Adobe Photoshop CS6.

Statistical analysis. Sample sizes were chosen based on previously published studies using similar conditions ${ }^{20}$. Infected animals were removed from the study based on previously established criteria of weight loss, which had to be between $5 \%$ and $20 \%$ between the day of infection and the $48 \mathrm{~h}$ timepoint, indicating a failed infection or requiring ethical sacrifice, respectively. Statistical analysis was performed using GraphPad Prism 9.0.0 for Windows (GraphPad Software, San Diego, CA, USA). Given data are indicated in the figure legends. Specific tests to determine statistical significance are noted in each sure legend. Correlation was acquired with linear regression best-fit, and the Pearson's correlation coefficient $r$ was calculated. $p$ values are depicted, with a value of $p<0.05$ considered significant. Collected PET and Biodistribution data passed the Shapiro-Wilk's normality test.

Reporting summary. Further information on research design is available in the Nature Research Reporting Summary linked to this article.

\section{Data availability}

Due to their large size, the raw imaging data that support the findings of this study are directly available from the corresponding authors upon reasonable request. Derived data have been compiled in the Source Data file provided with this paper. Any remaining data supporting the findings from this study are available from the corresponding author upon reasonable request.
Received: 3 April 2020; Accepted: 16 February 2021; Published online: 17 March 2021

\section{References}

1. Lescure, F.-X. et al. Clinical and virological data of the first cases of COVID-19 in Europe: a case series. Lancet Infect. Dis. 20, 697-706 (2020).

2. Li, Y. \& Xia, L. M. Coronavirus Disease 2019 (COVID-19): role of chest CT in diagnosis and management. Am. J. Roentgenol. 214, 1280-1286 (2020).

3. Yang, X. et al. Clinical course and outcomes of critically ill patients with SARS-CoV-2 pneumonia in Wuhan, China: a single-centered, retrospective, observational study. Lancet Respiratory Med. 8, 475-481 (2020).

4. Prattes, J. et al. Invasive pulmonary aspergillosis complicating COVID-19 in the ICU - A case report. Med. Mycol. Case Rep. 31, 2-5 (2021).

5. Meijer, E. F. J., Dofferhoff, A. S. M., Hoiting, O., Buil J. B. \& Meis J. F. Azoleresistant COVID-19-associated pulmonary aspergillosis in an immunocompetent host: a case report. J. Fungi 6, 79 (2020).

6. Brown, G. D. et al. Hidden killers: human fungal infections. Sci. Transl. Med. 4, 165 rv113 (2012).

7. Hope, W. W., Walsh, T. J. \& Denning, D. W. Laboratory diagnosis of invasive aspergillosis. Lancet Infect. Dis. 5, 609-622 (2005).

8. Segal, B. H. Aspergillosis. N. Engl. J. Med. 360, 1870-1884 (2009).

9. Garg, M. K., Gupta, P., Agarwal, R., Sodhi, K. S. \& Khandelwal, N. MRI: a new paradigm in imaging evaluation of allergic bronchopulmonary aspergillosis? Chest 147, e58-e59 (2015)

10. Loizidou, A., Aoun, M. \& Klastersky, J. Fever of unknown origin in cancer patients. Crit. Rev. Oncol. Hemat. 101, 125-130 (2016).

11. Vermeulen, E., Lagrou, K. \& Verweij, P. E. Azole resistance in Aspergillus fumigatus: a growing public health concern. Curr. Opin. Infect. Dis. 26, 493-500 (2013)

12. Thornton, C. R. Detection of the 'Big Five' mold killers of humans: aspergillus, fusarium, lomentospora, scedosporium and mucormycetes. In Advances in Applied Microbiology (eds. Geoffrey, G. \& Sima, S.) (Academic Press, 2019).

13. Thornton, C. R. Molecular imaging of invasive pulmonary aspergillosis using immunoPET/MRI: the future looks bright. Front. Microbiol. 9, 691 (2018).

14. Gordon, O., Ruiz-Bedoya, C. A., Ordonez, A. A., Tucker, E. W. \& Jain, S. K. Molecular imaging: a novel tool to visualize pathogenesis of infections in situ. Mbio 10, e00317-19 (2019).

15. Lupetti, A., Welling, M. M., Mazzi, U., Nibbering, P. H. \& Pauwels, E. K. J. Technetium-99m labelled fluconazole and antimicrobial peptides for imaging of Candida albicans and Aspergillus fumigatus infections. Eur. J. Nucl. Med Mol. I 29, 674-679 (2002).

16. Petrik, M. et al. $68 \mathrm{Ga}$-Siderophores for PET imaging of invasive pulmonary aspergillosis: proof of principle. J. Nucl. Med. 51, 639-645 (2010).

17. Petrik, M. et al. In Vitro and in vivo comparison of selected Ga-68 and Zr-89 labelled siderophores. Mol. Imaging Biol. 18, 344-352 (2016).

18. Thornton, C. R. Development of an immunochromatographic lateral-flow device for rapid serodiagnosis of invasive aspergillosis. Clin. Vaccin. Immunol. 15, 1095-1105 (2008)

19. Rolle, A. M. et al. ImmunoPET/MR imaging allows specific detection of Aspergillus fumigatus lung infection in vivo. Proc. Natl Acad. Sci. USA 113, E1026-E1033 (2016).

20. Davies, G. et al. Towards translational immunoPET/MR imaging of invasive pulmonary aspergillosis: the humanised monoclonal antibody JF5 detects aspergillus lung infections in vivo. Theranostics 7, 3398-3414 (2017).

21. Kovanda, L. L., Desai, A. V. \& Hope, W. W. Prognostic value of galactomannan: current evidence for monitoring response to antifungal therapy in patients with invasive aspergillosis. J. Pharmacokinet. Phar. 44, 143-151 (2017).

22. Wiederhold, N. P. et al. Interlaboratory and interstudy reproducibility of a novel lateral-flow device and influence of antifungal therapy on detection of invasive pulmonary aspergillosis. J. Clin. Microbiol. 51, 459-465 (2013).

23. Marr, K. A., Laverdiere, M., Gugel, A. \& Leisenring, W. Antifungal therapy decreases sensitivity of the Aspergillus galactomannan enzyme immunoassay. Clin. Infect. Dis. 40, 1762-1769 (2005).

24. Herbrecht, R. et al. Voriconazole versus amphotericin B for primary therapy of invasive aspergillosis. N. Engl. J. Med. 347, 408-415 (2002).

25. Lass-Flörl, C., Cuenca-Estrella, M., Denning, D. W. \& Rodriguez-Tudela, J. L. Antifungal susceptibility testing in Aspergillus spp. according to EUCAST methodology. Med. Mycol. 44, S319-S325 (2006).

26. Meletiadis, J., Leth Mortensen, K., Verweij, P. E., Mouton, J. W. \& Arendrup, M. C. Spectrophotometric reading of EUCAST antifungal susceptibility testing of Aspergillus fumigatus. Clin. Microbiol Infect. 23, 98-103 (2017).

27. Maertens, J. et al. Lateral flow assays for diagnosing invasive pulmonary aspergillosis in adult hematology patients: a comparative multicenter study. Med. Mycol. 58, 444-452 (2020). 
28. Mercier, T. et al. Diagnosing invasive pulmonary aspergillosis in hematology patients: a retrospective multicenter evaluation of a novel lateral flow device. J. Clin. Microbiol. 57, e01913-18 (2019).

29. Dichtl, K., Seybold, U., Ormanns, S., Horns, H. \& Wagener, J. Evaluation of a novel aspergillus antigen enzyme-linked immunosorbent assay. J. Clin. Microbiol. 57, e00136-19 (2019).

30. Skriba, A. et al. Early and non-invasive diagnosis of aspergillosis revealed by infection kinetics monitored in a rat model. Front. Microbiol. 9, 2356 (2018).

31. Marr, K. A. et al. Urine antigen detection as an aid to diagnose invasive aspergillosis. Clin. Infect. Dis. 67, 1705-1711 (2018).

32. Fernández-Cruz, A., Lewis, R. E. \& Kontoyiannis, D. P. How long do we need to treat an invasive mold disease in hematology patients? Factors influencing duration of therapy and future questions. Clin. Infect. Dis. 71, 685-692 (2020).

33. Lupetti, A., Welling, M. M., Pauwels, E. K. J. \& Nibbering, P. H. Detection of fungal infections using radiolabeled antifungal agents. Curr. Drug Targets 6, 945-954 (2005).

34. Jalilian, A., Sardari, D. \& Sardari, S. Application of radioisotopes in anti-fungal research and fungal disease studies. Curr. Med. Chem. Anti-Infective Agents 3, 325-338 (2004).

35. Pfister, J. et al. Hybrid imaging of aspergillus fumigatus pulmonary infection with fluorescent, 68Ga-labelled siderophores. Biomolecules 10, 168 (2020).

36. Petrik, M., Zhai, C., Haas, H. \& Decristoforo, C. Siderophores for molecular imaging applications. Clin. Transl. Imaging 5, 15-27 (2017).

37. Lee, M. H. et al. A novel, tomographic imaging probe for rapid diagnosis of fungal keratitis. Med. Mycol. 56, 796-802 (2018).

38. Hope, W. W. et al. The Initial $96 \mathrm{~h}$ of invasive pulmonary aspergillosis: histopathology, comparative kinetics of galactomannan and (1 -> 3)-beta-DGlucan, and consequences of delayed antifungal therapy. Antimicrob. Agents Chemother. 54, 4879-4886 (2010).

39. Neofytos, D. et al. Correlation between circulating fungal biomarkers and clinical outcome in invasive aspergillosis. PLOS ONE 10, e0129022 (2015).

40. Mercier, T., Guldentops, E., Lagrou, K. \& Maertens, J. Galactomannan, a surrogate marker for outcome in invasive aspergillosis: finally coming of age. Front. Microbiol. 9, 661 (2018).

41. Patel, R., Hossain, M. A., German, N. \& Al-Ahmad, A. J. Gliotoxin penetrates and impairs the integrity of the human blood-brain barrier in vitro. Mycotoxin Res. 34, 257-268 (2018).

42. Kruger, P. et al. Neutrophils: between host defence, immune modulation, and tissue injury. PLoS Pathog. 11, e1004651 (2015).

43. $\mathrm{Xu}, \mathrm{D}$. et al. Gr-1+ cells other than Ly6G+ neutrophils limit virus replication and promote myocardial inflammation and fibrosis following coxsackievirus B3 infection of mice. Front. Cell Infect. Microbiol. 8, 157 (2018).

44. Amich, J. et al. 3D light sheet fluorescence microscopy of lungs to dissect local host immune-Aspergillus fumigatus interactions. bioRxiv https://doi.org/ $10.1101 / 661157$ (2019).

45. Aimanianda, V. et al. Surface hydrophobin prevents immune recognition of airborne fungal spores. Nature 460, 1117-111 (2009).

46. Meletiadis, J. et al. Differential fungicidal activities of amphotericin B and voriconazole against Aspergillus species determined by microbroth methodology. Antimicrob. Agents Chemother. 51, 3329-3337 (2007).

47. Krishnan, S., Manavathu, E. K. \& Chandrasekar, P. H. A comparative study of fungicidal activities of voriconazole and amphotericin B against hyphae of Aspergillus fumigatus. J. Antimicrob. Chemother. 55, 914-920 (2005).

48. Espinel-Ingroff, A. In vitro fungicidal activities of voriconazole, itraconazole, and amphotericin B against opportunistic moniliaceous and dematiaceous fungi. J. Clin. Microbiol. 39, 954-958 (2001).

49. Borman, A. M. et al. MIC distributions and evaluation of fungicidal activity for amphotericin B, itraconazole, voriconazole, posaconazole and caspofungin and 20 species of pathogenic filamentous fungi determined using the CLSI broth microdilution method. J. Fungi 3, 27 (2017).

50. Lewis, R. E. et al. Epidemiology and sites of involvement of invasive fungal infections in patients with haematological malignancies: a 20-year autopsy study. Mycoses 56, 638-645 (2013).

51. Bizet, J., Cooper, C. J., Zuckerman, M. J., Torabi, A. \& Mendoza-Ladd, A. A bleeding colonic ulcer from invasive Aspergillus infection in an immunocompromised patient: a case report. J. Med. Case Rep. 8, 407 (2014).

52. Reichenberger, F., Habicht, J. M., Gratwohl, A. \& Tamm, M. Diagnosis and treatment of invasive pulmonary aspergillosis in neutropenic patients. Eur. Respiratory J. 19, 743-755 (2002).
53. Freise, A. C. \& Wu, A. M. In vivo imaging with antibodies and engineered fragments. Mol. Immunol. 67, 142-152 (2015).

54. Lother, J. et al. Human dendritic cell subsets display distinct interactions with the pathogenic mould Aspergillus fumigatus. Int. J. Med. Microbiol. 304, 1160-1168 (2014)

55. Mannheim, J. G. et al. Quantification accuracy and partial volume effect in dependence of the attenuation correction of a state-of-the-art small animal PET scanner. Phys. Med. Biol. 57, 3981-3993 (2012).

56. Susaki, E. A. et al. Advanced CUBIC protocols for whole-brain and wholebody clearing and imaging. Nat. Protoc. 10, 1709-1727 (2015).

\section{Acknowledgements}

We are grateful to Walter Ehrlichmann and Dominik Seyfried for the radiotracer production. We thank Maren Harant, Linda Schramm, and Nathalie Mucha for their expert technical assistance, Simon Freisinger for his experimental help, Julia Mannheim, Johannes Schwenck, Stefan Wiehr, and Anna-Maria Wild for their scientific input, and the Imaging Center Essen (IMCES) for supporting all optical imaging. We thank Sven Krappmann for providing the A. fumigatus ${ }^{\text {tdTomato }}$ strain. We acknowledge funding by the Ministry of Culture and Science of North Rhine-Westphalia, the Governing Mayor of Berlin including Science and Research, and the Federal Ministry of Education and Research to M.G. This work was supported by the European Union Seventh Framework Programme FP7/2007-2013 under Grant 602820, and the Werner Siemens Foundation.

\section{Author contributions}

Conceptualization and planning: S.H., M.H., C.R.T., B.J.P., M.G., and N.B.; Experi mentation: S.H., A.M., L.B., F.N., I.G.M., A.K., M.H., and N.B.; Data analysis: S.H., and N.B.; Manuscript and figures preparation: S.H., A.H., I.G.M., A.K., M.H., C.R.T., B.J.P., M.G., and N.B.

\section{Funding}

Open Access funding enabled and organized by Projekt DEAL.

\section{Competing interests}

C.R.T. is director of ISCA Diagnostics Ltd. The remaining authors declare no competing interests.

\section{Additional information}

Supplementary information The online version contains supplementary material available at https://doi.org/10.1038/s41467-021-21965-z.

Correspondence and requests for materials should be addressed to M.G. or N.B.

Peer review information Nature Communications thanks David Denning and the other, anonymous, reviewer(s) for their contribution to the peer review of this work. Peer reviewer reports are available.

Reprints and permission information is available at http://www.nature.com/reprints

Publisher's note Springer Nature remains neutral with regard to jurisdictional claims in published maps and institutional affiliations.

Open Access This article is licensed under a Creative Commons Attribution 4.0 International License, which permits use, sharing, adaptation, distribution and reproduction in any medium or format, as long as you give appropriate credit to the original author(s) and the source, provide a link to the Creative Commons license, and indicate if changes were made. The images or other third party material in this article are included in the article's Creative Commons license, unless indicated otherwise in a credit line to the material. If material is not included in the article's Creative Commons license and your intended use is not permitted by statutory regulation or exceeds the permitted use, you will need to obtain permission directly from the copyright holder. To view a copy of this license, visit http://creativecommons.org/ licenses/by/4.0/.

(C) The Author(s) 2021 